\title{
DER EINZUG DER EDV IM ÖSTERREICHISCHEN BIBLIOTHEIKSWESEN AM BEISPIEL DER UNIVERSITÄTSBIBLIOTHEK GRAZ
}

\section{von Karin Lackner und Lisa Schilhan}

Zusammenfassung: Durch den Einsatz von EDV-Systemen kam es ab den 1970er Jahren zu einem radikalen Wandel in der Benutzung und Verwaltung von Universitätsbibliotheken. Die Universitätsbibliothek Graz war die erste Bibliothek in Österreich, die ein elektronisches Bibliothekssystem entwickelte und einsetzte, womit sie zu den Vorreitern in Europa zählte. Dieser Artikel liefert einen historischen Überblick über die Anfänge, die Entwicklung und Verbreitung der elektronischen Bibliothekssysteme im Allgemeinen sowie an der Universitätsbibliothek Graz im Speziellen. Vorgestellt werden die im Lauf der Jahrzehnte an der UB Graz eingesetzten Bibliothekssysteme GRIBS, EMILE, FBInfo, BIBOS, ALEPH und ALMA sowie die Entwicklung von den ersten Online- über die CD-ROM-Datenbanken bis hin zum modernen DatenbankRetrieval.

Schlagworte: Bibliotheksautomatisation; Universitätsbibliothek Graz; Geschichte

\section{THE APPLICATION OF EDP IN AUSTRIAN LIBRARIES USING THE EXAMPLE OF THE UNIVERSITY OF GRAZ LIBRARY}

Abstract: The use of EDP systems brought about a radical change in the use and management of university libraries from the 1970s onwards. The library of the University of Graz was one of the first libraries in Europe, as well as the first library in Austria, to develop and deploy an electronic library system. This article provides a historical overview of the beginnings, development, and distribution of electronic library systems in general and at the University of Graz in particular. It presents the library systems GRIBS, EMILE, FBInfo, BIBOS, ALEPH, and ALMA, which have been used over the decades, as well as the development from the first online databases to $C D$ ROM databases and modern database retrieval.

Keywords: library automation; Graz University Library; history

DOI: https://doi.org/10.31263/voebm.v74i2.6395

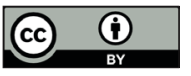

Dieses Werk ist - exkl. einzelner Logos und Abbildungen - lizenziert unter einer Creative-Commons-Lizenz Namensnennung 4.0 International-Lizenz 
Methode: Der vorliegende Text beruht auf mehreren Interviews mit Frau Dipl.-Ing. in Burghild Schubert, geführt von MMag. ${ }^{a}$ Karin Lackner und Dr. ${ }^{\text {in }}$ Lisa Schilhan zwischen Dezember 2020 und Oktober 2021. Frau Schubert wurde 1977 speziell für die Einführung der EDV an der Universitätsbibliothek Graz in der Abteilung „Information" angestellt und war in ihrer über 40jährigen Dienstzeit für die Betreuung und Weiterentwicklung der EDV und Bibliothekssysteme an der UB Graz zuständig. Von Ende 2008 bis zu ihrer Pensionierung im Frühjahr 2019 leitete sie die Abteilung.

Neben den Interviews wurden die Veröffentlichungen von Frau Schubert im Zusammenhang mit der Einführung von EDV an der UB Graz sowie die Jahresberichte der UB Graz aus den Berichtsjahren 1977 bis 2017 herangezogen. Die Berücksichtigung weiterer Literatur wurde bewusst gering gehalten, da der Fokus des Textes auf den Entwicklungen an der UB Graz und damit in erster Linie aufden persönlichen Erinnerungen von Frau Schubert sowie ergänzend auf den Jahresberichten der UB Graz liegt.

\section{Einleitung: Bibliothekarische Arbeit vor Einführung der EDV}

Die systematische Erfassung von Bibliotheksbeständen erfolgte seit dem Mittelalter mittels so genannter Bandkataloge. Die Erwerbungen einer Bibliothek wurden handschriftlich und in alphabetischer Reihenfolge eingetragen, wobei zwischen den Einträgen Platz für nachträgliche Ergänzungen freigelassen wurde. Im 19. Jahrhundert wurden die Bandkataloge durch die Zettelkataloge abgelöst. Zettelkataloge bestanden aus Katalogkästen, in denen die Katalogkarten - je eine pro Werk - alphabetisch geordnet in Schubladen aufbewahrt wurden. Zettelkataloge boten gegenüber Bandkatalogen den großen Vorteil, beliebig erweiterbar zu sein.

Für jedes Werk eine Katalogkarte händisch bzw. später mit Schreibmaschine auszufüllen, war allerdings sehr zeitaufwändig und musste in jeder Bibliothek, die dieses Werk anschaffte, durchgeführt werden. Eine erste Arbeitserleichterung war daher ein neu eingeführter Service der Library of Congress: Bibliotheken in den USA konnten dort für ihre neu erworbenen Werke gedruckte Katalogkarten anfordern. Lediglich lokale Notizen mussten in den einzelnen Bibliotheken weiterhin händisch angebracht bzw. getippt werden. ${ }^{1}$

Die EDV-Voraussetzungen für die Entwicklung elektronischer Bibliothekssysteme wurden erst in den 1960er und 1970er Jahren geschaffen. Das „Arpanet“, der Vorläufer des Internets, ging im Herbst 1969 in den USA in Betrieb und vernetzte Großrechenanlagen von Universitäten und Forschungseinrichtungen. Es hatte zunächst hauptsächlich den Zweck eines Kommunikationsmittels, die wichtigste Anwendung war die E-Mail. Europa war nicht an das Arpanet angeschlossen. ${ }^{2}$ 
Zwar wurden bereits damals Zeitschriftenartikel in Datenbanken elektronisch erfasst, die Abfragen waren jedoch nur als batch job ${ }^{3}$ möglich, nicht online.

Zeitgleich wurden ab Ende der 1960er Jahre die ersten elektronischen Bibliothekssysteme entwickelt. Das Ziel war die Entwicklung einer Software, mit der alle Geschäftsgänge einer Bibliothek wie Erwerbung, Titelerfassung und Entlehnung abgewickelt werden konnten.

\section{Die 1970er und 1980er Jahre}

\subsection{Erste EDV-gestützte Bibliothekssysteme: Der Online Union Catalog}

1967 wurde in den USA OCLC (damals: Ohio College Library Center, heute: Online Computer Library Center) gegründet. 1971 führte OCLC das erste Online-Katalogisierungssystem ein, bei dem Bibliothekar:innen der teilnehmenden Bibliotheken ihre lokalen Bestände zentral erfassen sowie bereits erfasste Datensätze aller teilnehmenden Bibliotheken in Echtzeit suchen, nutzen und bearbeiten konnten. Der Online Union Catalog von OCLC war damit der weltweit erste interaktive und dynamische Verbundkatalog. OCLC produzierte für jede Bibliothek die benötigten Katalogkarten und sandte diese zu. Vor Ort konnten die Karten dann in die bestehenden Zettelkataloge einsortiert werden. ${ }^{4}$ Der Online Union Catalog ist heute unter dem Namen „WorldCat“ bekannt.

\subsection{Situation in Europa, Österreich und an der UB Graz}

Amerikanische Bibliotheken hatten mit dem Online Union Catalog von OCLC bereits früh ein System zur zentralen Erfassung von Datensätzen, jedoch kein System, mit dem sie Magazinsentlehnungen verwalten konnten. Ein solches System war in Amerika nicht notwendig, da die dortigen Bibliotheken Freihandbibliotheken waren, bei denen die Benutzer:innen selbst zum Regal gehen und sich das gewünschte Buch herausnehmen konnten. Damit hatten amerikanische Bibliotheken jenes Problem nicht, das in Europa vordringlich war: Die meisten größeren Bibliotheken in Europa waren und sind Magazinbibliotheken. Um ein Buch aus dem Magazin zu entlehnen, füllten die Benutzer:innen einen Entlehnschein aus, mit dem ein:e Bibliothekar:in das Buch im Magazin suchte. Wenn das Buch entlehnt war - was insbesondere bei Büchern, die in Lehrveranstaltungen empfohlen und dadurch von vielen Studierenden bestellt wurden, häufig vorkam -, wurde an der UB 
Graz ein so genannter „N.E.-Schein“ (N.E. - nicht eingestellt) ausgefüllt. Der Gang ins Magazin war also bei vielen Bestellungen umsonst. Diese Leerläufe bedeuteten einen hohen Zeitaufwand für das Personal und kosteten unnötige Arbeitszeit. Auch für die Studierenden war es frustrierend, einen Entlehnschein auszufüllen und am nächsten Tag zu erfahren, dass das Buch bereits entlehnt war. Insbesondere für die großen Magazinbibliotheken wurde daher ein System benötigt, in dem die Entlehnungen erfasst werden konnten und vorab anzeigt wurde, ob sich ein Buch überhaupt am Standort befand.

Bereits seit den 1960er Jahren hatte es in Europa Bestrebungen gegeben, eine Software zu entwickeln, mit der alle Geschäftsgänge einer Bibliothek zentral verwaltet und einmal angelegte Datensätze von verschiedenen Modulen genutzt werden konnten (so genanntes „integriertes Bibliothekssystem"). Bislang waren für die verschiedenen Arbeitsschritte stets unterschiedliche Systeme verwendet und Datensätze daher mehrfach erfasst worden. Die erste europäische Bibliothek, die ein EDV-gestütztes Bibliothekssystem besaß, war jene der ETH Zürich mit dem selbst entwickelten System ELAS (Elektronisches Ausleihkontrollsystem, siehe Abb. 1) ${ }^{5}$.

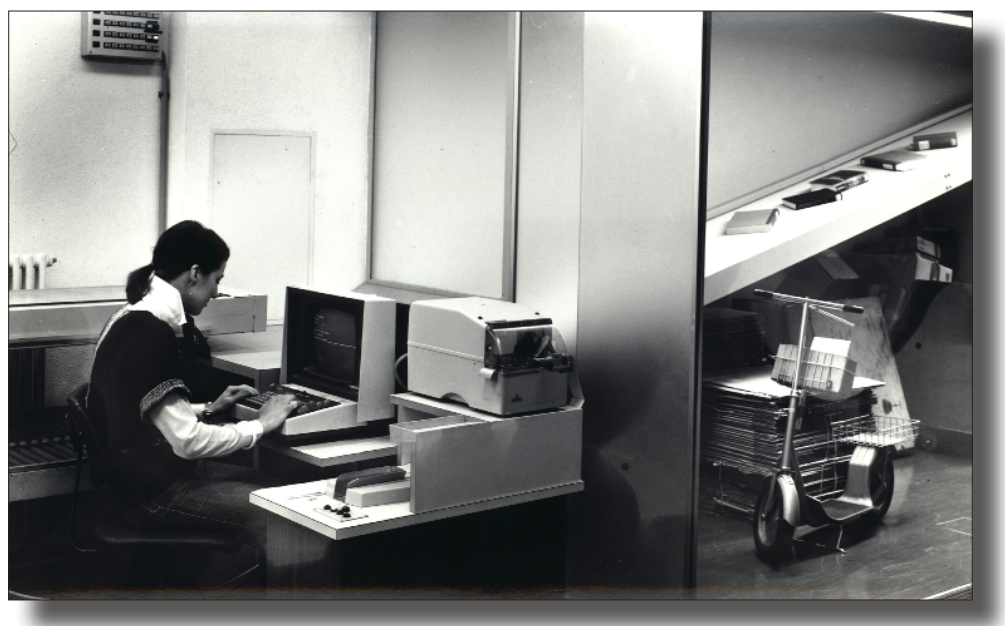

Abb. 1: ETH Zürich, Elektronisches Ausleihkontroll-System ELAS (1975), http://doi. org/10.3932/ethz-a-000013120, CC BY-SA 4.0.

\subsection{Modellprojekt „Automatisierung der Entlehnverbuchung an der Universi- tätsbibliothek Graz"“}

Der damalige Leiter der UB Graz, Hofrat Dr. Franz Kroller, war sehr an einem EDV-gestützten Bibliothekssystem interessiert, um unnötige Wege 
des Personals ins Magazin zu vermeiden. Neben der Erfassung von Entlehnungen sollten mit dem System außerdem Bestellungen, Vormerkungen und Mahnungen zentral verwaltet werden können.

Zu diesem Zweck wurde das Modellprojekt „Automatisierung der Entlehnverbuchung an der Universitätsbibliothek Graz" initiiert. Nach einer mehrjährigen Vorbereitungsphase fiel 1977 der Projektstart mit der Lieferung der erforderlichen EDV-Geräte und Programme, darunter ein Honeywell Bull Rechner, der an der Wiener Planungsstelle für das wissenschaftliche Bibliothekswesen ${ }^{6}$ aufgestellt wurde. ${ }^{7}$

Für die Einführung eines solchen Systems wurde 1977 an der UB Graz eine eigene Stelle für eine:n Angehörige:n des Höheren Dienstes mit abgeschlossenem Studium der Technischen Mathematik geschaffen, ${ }^{8}$ da der Bibliotheksleiter Herr Kroller darauf Wert legte, jemanden mit fundierten EDV-Kenntnissen im Haus zu haben. Der Posten wurde mit der damals 23jährigen Burghild Schubert besetzt. Herr Kroller hatte keine Bedenken, im damals noch sehr männerdominierten EDV-Bereich eine Frau einzusetzen - im Gegensatz zu vielen Unternehmen, die Vorbehalte gegenüber Frauen im EDV-Bereich hatten. ${ }^{9}$

Da die Bibliothek der ETH Zürich mit ELAS bereits ein EDV-gestütztes Bibliothekssystem besaß und darüber hinaus wie die UB Graz eine Magazinbibliothek war, reisten Herr Kroller und Frau Schubert zur ETH Zürich, um sich dieses System vor Ort anzusehen und gegebenenfalls zu übernehmen.

Das System ELAS hätte für die UB Graz sehr gut gepasst, konnte jedoch aus mehreren Gründen nicht übernommen werden. So stand an der ETH Zürich ein deutlich größeres Rechenzentrum für die personalintensive Systembetreuung zur Verfügung, als an der Universität Graz vorhanden war. Eine Hosting-Lösung war ebenfalls nicht möglich, da die technischen Möglichkeiten damals begrenzt waren und eine Standleitung von Graz nach Zürich zu lang und teuer gewesen wäre (die längste Standleitung der UB Graz war jene nach Wien). Daher konnte ELAS an der UB Graz aufgrund der fehlenden personellen und technischen Infrastruktur nicht übernommen werden. In Kooperation mit der Planungsstelle für wissenschaftliches Bibliothekswesen wurde infolgedessen ein eigenes System für die UB Graz entwickelt. Damit war die UB Graz nach der ETH Zürich die zweite Bibliothek Europas, die über eine EDV-gestützte Magazinsentlehnung verfügte, und zugleich die erste Bibliothek Österreichs, die EDV einführte. Das System der UB Graz, das im Folgenden genauer vorgestellt wird, wurde auch von mehreren anderen Institutionen in Österreich übernommen. 


\subsubsection{Entwicklung von GRIBS}

Das so genannte Grazer Integrierte Bibliothekssystem (GRIBS) wurde als Pilotprojekt der Wiener Planungsstelle für das wissenschaftliche Bibliothekswesen unter maßgeblicher Beteiligung von Burghild Schubert entwickelt.

Zu diesem Zweck wurde im Lauf des Jahres 1978 ein Pflichtenheft erarbeitet. ${ }^{10}$ Frau Schubert erhob hierfür die Bedarfe und Anforderungen der UB Graz und skizzierte diese detailliert, inklusive Flussdiagrammen. Die Planungsstelle in Wien setzte die Anforderungen anschließend in Code um.

Für die Entwicklung von GRIBS bediente man sich der Tatsache, dass die Bücher nach numerus currens aufgestellt waren. Zunächst wurden vier Formatgruppen mit den römischen Zahlen I bis IV festgelegt und den Formaten Signaturenblöcke zugeordnet. Die Vergabe der Signaturen erfolgte anschließend mit fortlaufendem numerus currens. Daneben wurden Lehrbuchsignaturen, Sonderstandorte (z.B. Semesterhandapparat) und die so genannten Spiegelsignaturen ${ }^{11}$ erfasst. Jede Signatur war bestellbar und bekam einen Status (z.B. Monographie, Spiegelsignatur, Zeitschrift/ Serie ohne Spiegel) zugeordnet. Der Status war für die Bestellung wichtig. Daneben gab es verschiedene Benutzerkategorien (z.B. wissenschaftliches Personal, nichtwissenschaftliches Personal, Studierende, Dissertanten, Angestellte der UB, Allgemeine Benutzer, Buchbinder, Fernleihe, Schaukasten $)^{12}$. Die Entlehnfristen, die Anzahl der maximal entlehnbaren Werke sowie die Befreiung von etwaigen Mahngebühren hing vom jeweiligen Benutzerstatus ab.

\subsubsection{Inbetriebnahme und Funktionsweise von GRIBS}

Für die Inbetriebnahme von GRIBS wurde 1979 auch an der UB Graz ein Honeywell-Bull-Rechner angeschafft (siehe Abb. 2), der im Keller der Hauptbibliothek aufgestellt wurde. Die Datensicherung erfolgte auf Disketten und Platten. Die Disketten wurden mit der Bahn nach Wien geschickt, um auch dort eine Sicherheitskopie abzulegen. ${ }^{13}$ Ab 1986 erfolgte die Datensicherung ausschließlich auf Platten. ${ }^{14}$

Die Inbetriebnahme von GRIBS erfolgte gestaffelt - zunächst 1980 für die Lehrbuchsammlung mit einem Probebetrieb an vier Bestellterminals. ${ }^{15}$ Diese Terminals boten noch keine grafische Benutzeroberfläche, die Bestellung konnte in der untersten Zeile des Bildschirms eingegeben werden.

Die Umstellung auf die automatisierte Entlehnung machte auch neue Bibliotheksausweise erforderlich, die nun maschinenlesbar und mit einem 
Foto des bzw. der Benutzer:in ausgestattet waren. ${ }^{16}$ Dazu mussten zunächst die Benutzerdaten in GRIBS erfasst werden. Zu diesem Zweck wurden die von der Universität erfassten Benutzerdaten auf einen Schlag vom EDV-System der Universität nach GRIBS überspielt - eine große Leistung, denn die UB war damals noch kein Teil der Universität, sondern dem Ministerium unterstellt. ${ }^{17}$

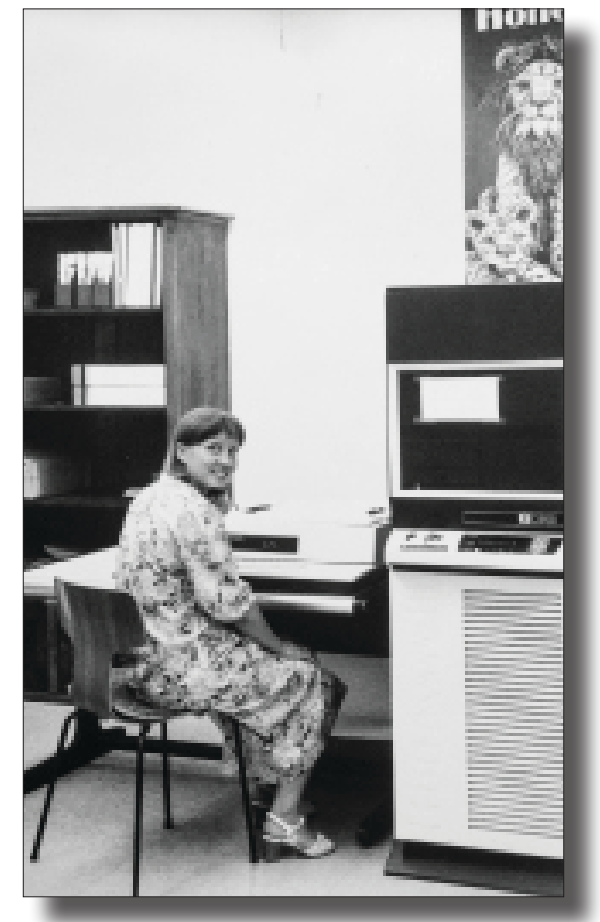

Abb. 2: Dipl.-Ing. ${ }^{\text {in }}$ Burghild Schubert am Honeywell Bull Rechner (Quelle: Jahresbericht der UB Graz für das Jahr 1980).

Da in GRIBS keine Recherchemöglichkeit bestand, musste allerdings weiterhin der Zettelkatalog oder der Mikrofiche-Katalog herangezogen werden, um Literatur zu suchen und die Signaturen zu den benötigten Werken herauszufinden.

Mit dem neuen Entlehnsystem waren sowohl die Bibliothekar:innen als auch die Studierenden von Beginn an sehr zufrieden. Auch mehrere dezentrale Standorte der UB sowie andere Grazer Universitätsbibliotheken zeigten sich an dem System sehr interessiert. ${ }^{18} \mathrm{Nach}$ der Lehrbuchsammlung sollte in einem nächsten Schritt auch die Bestellung und Entlehnung des Magazins- 
bestandes über GRIBS erfolgen. Nach Abschluss der aufwändigen Vorbereitungen und der Testphase erfolgte am 7. Juli 1983 die Inbetriebnahme von GRIBS für die automatisierte Magazinsentlehnung. Im Vorfeld wurden auf Basis der Entlehnstatistik die am häufigsten entlehnten Magazinsbücher im Magazin etikettiert. Die anderen Bücher wurden bei Entlehnung an der Ausleihe etikettiert. Bestand noch keine Konkordanz zwischen Etikett und Signatur, wurde sie bei der Entlehnung durch das Einscannen der Ausweisnummer und des Etiketts erstellt. Gleichzeitig wurde die Entlehnung verbucht. ${ }^{19}$

Mit den neuen Ausweisen konnten die Benutzer:innen die Bestellung selbst am Terminal durchführen (siehe Abb. 3), indem sie mit einem Lesestift ihr Benutzeretikett einlasen, die gewünschte(n) Signatur(en) eingaben und danach das Benutzeretikett nochmals einlasen. Verlängerungen und Vormerkungen konnten in gleicher Weise durchgeführt werden. ${ }^{20}$

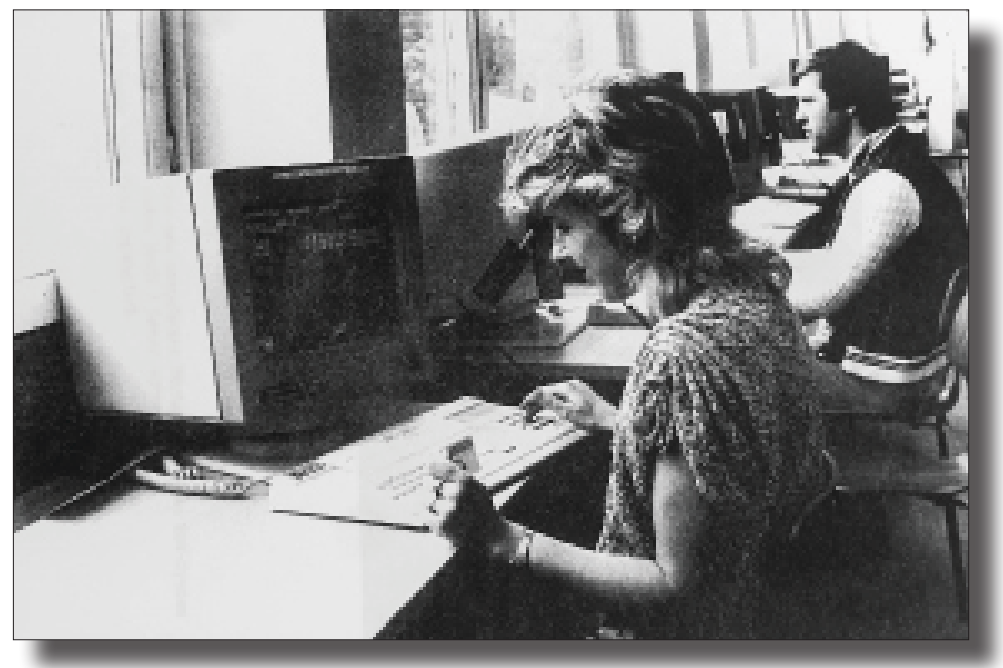

Abb. 3: Benutzer:innenterminals im Katalograum (Quelle: Jahresbericht der UB Graz für das Jahr 1984, S. 47).

Mit Wintersemester 1983 waren zehn Terminals für die Benutzer:innen in Betrieb. Auch der Ausleihschalter erhielt drei Arbeitsterminals mit Druckern, die Lesesaalausleihe ein Terminal. Einige weitere Abteilungen erhielten ebenfalls je ein Terminal. ${ }^{21}$ Am Ende des Jahres 1986 waren bereits 36 Terminals angeschlossen. ${ }^{22}$

Mit GRIBS konnten Bestellungen, Entlehnungen, Verlängerungen und Mahnungen verwaltet werden. Eine Entlehnstatistik konnte ebenfalls erstellt werden. 
Mit dem Umstieg auf die automatisierte Ausleihe konnten die Entlehnungen aus dem Magazin sprunghaft verdreifacht, jene aus der Lehrbuchsammlung mehr als verdoppelt werden. ${ }^{23} 1986$ erfolgte die Koppelung des Honeywell Bull mit dem Rechner des EDV-Zentrums der Universität Graz, sodass von allen Instituten, die an das EDV-Zentrum angeschlossen waren, direkt Bestellungen, Vormerkungen und Verlängerungen in GRIBS vorgenommen werden konnten. ${ }^{24}$

Die automatisierte Ausleihe war durch die automatische Mahnung und Sperre, einen einfachen Ausleih- und Rückgabevorgang sowie den Wegfall unnötiger Personalwege nicht nur eine deutliche Arbeitserleichterung für die Bibliothekar:innen, sondern bot auch den Benutzer:innen zahlreiche Vorteile wie Sofortauskunft über den Zeitpunkt der Verfügbarkeit eines Werkes sowie den Kontostand (Mahngebühren), Wegfall des händischen Ausfüllens von Bestellscheinen und schnelle Abfertigung. ${ }^{25} \mathrm{Im}$ Fall eines Systemausfalls war weiterhin die Ausleihe per händisch auszufüllendem Bestellschein sowie die Rückgabe von Büchern möglich, nicht jedoch die Verlängerung oder Vormerkung von Werken. ${ }^{26}$

\subsubsection{GRIBS-Erweiterung: Einführung der Kurztitelerfassung und der Recher- chefunktion mit GRIBSinfo}

GRIBS beruhte in erster Linie auf der Signaturerfassung, da die Bücher von den Bibliotheksmitarbeiter:innen über ihre Signatur ausgehoben und rückgestellt wurden, nicht über die Titel, die anfangs daher auch nicht erfasst wurden. Dies führte dazu, dass Benutzer:innen zwar Mahnungen erhielten, wenn die Entlehnfrist eines Werkes ablief, jedoch nur die Information zur Signatur erhielten, keine Titelinformationen. Aufgrund von Beschwerden wurde 1985 eine Titelerfassung eingeführt, jedoch stand in GRIBS dafür nur eine einzige Bildschirmzeile und damit eine geringe Zeichenanzahl zur Verfügung, sodass die meisten Titel nicht vollständig erfasst werden konnten, da Autorenname, Titel und Erscheinungsjahr in einer Zeile eingetragen werden mussten. Die Vorgaben lauteten daher, den Nachnamen sowie abgekürzten Vornamen des Autors bzw. der Autorin und den abgekürzten Titel zu erfassen, wobei mindestens ein sinnstiftendes Titelwort ausgeschrieben sein musste. ${ }^{27}$

Die Kurztitelerfassung wurde für alle Neuerwerbungen sowie rückwirkend bei der Rückgabe entliehener älterer Werke durchgeführt. Eine systematische Rückerfassung erfolgte nicht, sodass Werke, die nicht entlehnt wurden, nicht mit einem Kurztitel versehen wurden.

Neben der Titelerfassung gab es auch den Wunsch nach einer Recherchierbarkeit der im System enthaltenen Titel. Um diesen neuen An- 
forderungen Rechnung zu tragen, wurde GRIBSinfo 1987 vom EDV-Zentrum der Universität Graz entwickelt und im November 1987 mit über 150.000 Kurztiteln in Betrieb genommen. ${ }^{28}$ Recherchiert werden konnte nach Autor:in, Titelwort und Signatur sowie im Titelindex. Auch eine kombinierte Suche aus Autor:in und Titelwort war möglich. Für die Suche konnte auch eine Rechts- und Linkstrunkierung verwendet werden (siehe Abb. 4). Auf GRIBSinfo konnte auch von außerhalb der Universität zugegriffen werden, sodass eine Recherche unabhängig von den Öffnungszeiten der Bibliothek möglich war.

Ursprünglich war kein Datenaustausch von GRIBS nach GRIBSinfo geplant, sodass der Datenbestand in GRIBSinfo sukzessive veraltet wäre. Daher wurde nachträglich eine wöchentliche Datenaktualisierung eingeführt.

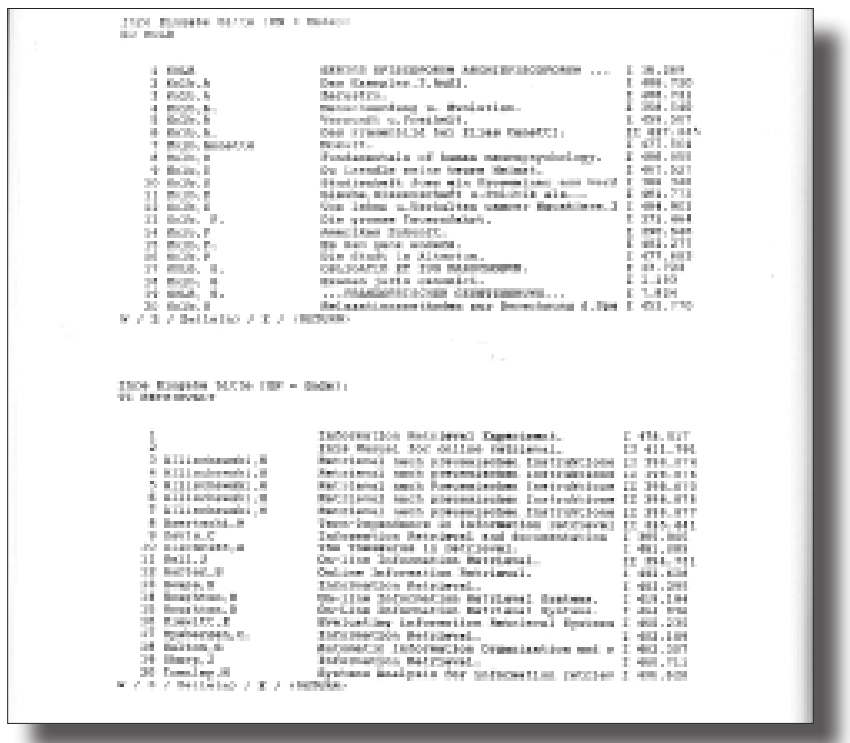

Abb. 4: Beispiele für eine Autorensuche und eine Titelsuche mit Trunkierung in GRIBSinfo (Quelle: Jahresbericht der UB Graz für das Jahr 1987, S. 30).

\subsubsection{Verbreitung von GRIBS}

Die Entwicklung von GRIBS fand zwar in Graz statt, jedoch bestand von Anfang an der Plan, dass GRIBS auch von anderen Bibliotheken übernommen werden kann. Dies geschah auch teilweise. So übernahmen die Bibliotheken der Universitäten Wien, Linz und Salzburg sowie der TU Wien das System. Es war allerdings in erster Linie auf die Bedürfnisse der UB Graz zugeschnitten, 
für welche die Entlehnung eine wichtige Rolle spielte. Weitere geplante Funktionen wie ein elektronisches Erwerbungsmodul wurden letztendlich nicht entwickelt, und auch die Titelerfassung war, wie bereits erwähnt, nur unzureichend möglich. Während die UB Graz mit GRIBS sehr zufrieden war, war das System daher für andere Bibliotheken nicht immer optimal einsetzbar.

\subsection{Weitere Systeme und Services an der UB Graz}

\subsubsection{EMILE und FBInfo}

GRIBS wurde nur in der Hauptbibliothek betrieben. Die Fachbibliotheken setzten GRIBS nicht ein, da die dezentralen Standorte der UB damals weitgehend unabhängig von der Hauptbibliothek arbeiteten und die technischen Möglichkeiten, die den Betrieb eines zentralen Systems an mehreren dezentralen Standorten betrafen, noch nicht so ausgefeilt waren wie heutzutage. Daher wurden an mehreren Standorten eigene Systeme entwickelt, die den Bedürfnissen der jeweiligen Teilbibliotheken angepasst waren.

Am Institut für Erziehungswissenschaft wurde ab 1989 parallel zu GRIBS das auf der Bibliothekssoftware Allegro basierende Bibliotheksverwaltungssystem EMILE für Erfassung, Entlehnung und Mahnwesen entwickelt. Es wurde an den Fachbibliotheken für Romanistik, Slawistik, Dolmetscher und Übersetzer sowie Erziehungswissenschaften verwendet. Da die jeweiligen Institute vor Einführung von EMILE unterschiedliche Datenverwaltungssysteme wie dBase verwendet hatten, wurden die Datensätze aus diesen Systemen in EMILE übernommen. ${ }^{29}$

An der Bibliothek der Rechts-, Sozial- und Wirtschaftswissenschaften (RESOWI) wurde in Zusammenarbeit mit dem EDV-Zentrum ab 1989 das Bibliothekssystem FBInfo ${ }^{30}$ entwickelt. Sowohl FBInfo als auch EMILE waren zunächst ein reines Titelerfassungssystem ohne Bestellkomponente. Bei EMILE wurde später die automatisierte Freihandentlehnung eingeführt (jedoch keine Magazinsentlehnung). Beide Systeme hatten gegenüber GRIBS den Vorteil, dass die Titeleingabe nicht auf eine geringe Zeichenanzahl beschränkt war.

\subsubsection{UBIS}

Zeitgleich mit GRIBS wurde an der UB Graz 1979 ein neuer Informationsdienst eingerichtet, die „Universitätsbibliothek Informationsvermittlungsstelle“ (UBIS), für die auch Dipl.-Ing. Schubert zeitweise arbeitete. UBIS 
war mit einem Bildschirmterminal, einem Drucker, einem Modem 300 und einem Datentelefon ausgestattet und unterstützte die Benutzer:innen bei der Beschaffung von Literaturstellen und Originaldokumenten - sowohl auf konventionelle als auch maschinelle Weise. UBIS hatte Zugriff auf die Datenzentrale in Palo Alto (Kalifornien); zu den meistgenutzten Datenbanken zählten 1979 MedLine mit 34\% und die Chemical Abstracts mit 13,6\%. ${ }^{31}$

Viele Anfragen bei UBIS bezogen sich darauf, wie oft ein:e Forscher:in zitiert wurde. UBIS führte damals bereits bibliometrische Analysen durch. Auch auf den Impact Factor bezogen sich viele Anfragen. Dieser war ausschließlich über den Science Citation Index ( $\mathrm{SCl}$, siehe Kap. 1.5.) suchbar. Die Impact Factors wurden von den Mitarbeiter:innen der UB Graz im SCI recherchiert und in Hefte gebunden. Insbesondere für die Mediziner:innen spielte der Impact Factor bereits in den 1980er Jahren eine wichtige Rolle, wenn sie sich auf eine Primararztstelle oder eine Professur bewarben.

UBIS lief parallel zum so genannten Katalogdienst bzw. der Katalogauskunft, bei der die Benutzer:innen bei der Literatursuche im Zettelkatalog und später im Onlinekatalog unterstützt wurden.

Mit der zunehmenden Verfügbarkeit der Datenbanken im Internet (siehe Kap. 3.1.), die das eigenständige Recherchieren der Benutzer:innen ermöglichten, und sinkender Zahl an Datenbanken, die nur für Bibliothekar:innen zugänglich waren, nahm der Bedarf an Recherchen durch UBIS kontinuierlich ab, sodass die Informationsvermittlungsstelle nach und nach in der Katalogauskunft, die später zu „Fachinformation“ umbenannt wurde, aufging. Die Fachinformation übernahm die von UBIS geleistete vertiefende Hilfestellung zu Datenbankrecherchen. Sie war und ist darüber hinaus als fachlicher Auskunftsdienst zuständig für alle Anfragen rund um die Benützung der Bibliothek und der physischen sowie elektronischen Bestände in unikat, dem Suchportal der UB Graz, sowie für Beratung zu Recherche und Suchstrategien.

\subsection{3. ÖZDB}

Parallel zu GRIBS wurde ab 1982 an der Wiener Planungsstelle für das wissenschaftliche Bibliothekswesen an der Österreichischen Nationalbibliothek die Österreichische Zeitschriftendatenbank (ÖZDB) als Bestandsnachweis für nationale und internationale Zeitschriften entwickelt ${ }^{32}$ und 1984 eingerichtet. In der ÖZDB konnte man Datensätze nicht selbst erfassen, sondern musste neue Einträge an die ÖZDB melden.

1985 erhielt die UB Graz einen Anschluss an den Rechner der Planungsstelle, sodass nun eine direkte Online-Abfrage der ÖZDB möglich war. ${ }^{33}$ 
Die ÖZDB wurde vom GRIBS-Nachfolgesystem BIBOS (siehe Kap. 2.2.) nicht übernommen, sodass sowohl zentral in der ÖZDB als auch von den einzelnen Bibliotheken in BIBOS Zeitschriftendatensätze erfasst wurden. Da die beiden Systeme parallel liefen, die Daten jedoch nicht gegeneinander abgeglichen wurden, liefen die Datenbestände mit der Zeit auseinander. Mit Einführung des BIBOS-Nachfolgesystems Aleph (siehe Kap. 3.2.) wurde versucht, die beiden Systeme zu vereinen, was zu Problemen bei der Datenmigration führte.

\subsection{Datenbankrecherche in den 1970er und 1980er Jahren}

Bibliothekskataloge und OPACs ${ }^{34}$ wiesen nur selbstständige Werke wie Bücher und Zeitschriften nach. Unselbstständige Werke wie Zeitschriftenartikel wurden in gedruckten Bibliographien nachgewiesen. Der Vorläufer der Datenbanken war der Science Citation Index (SCI) in Buchform. Er war alphabetisch nach den Autor:innen und innerhalb eines Autorennamens alphabetisch nach Titel sortiert, entweder monatsweise, jahresweise oder 5-jahresweise. Neben der Suche nach Autorennamen war auch eine Titelsuche möglich. Hierfür stellte der SCI den so genannten Permuterm Subject Index (PSI) ${ }^{35}$ zur Verfügung. Der PSI enthielt nicht nur eine nach dem ersten Titelwort alphabetisch geordnete Titelliste, sondern auch je eine nach dem zweiten und dritten Titelwort geordnete Liste. Diese permutierte Suche, mit der nicht nur nach dem ersten, sondern nach den ersten drei Titelwörtern gesucht werden konnte, war für die Literaturrecherche ausgesprochen hilfreich, wenn der genaue Titel und damit das erste Titelwort nicht bekannt waren. Neben dem SCl und ERIC zählten Medline und die Physical Abstracts zu den ersten OnlineDatenbanken.

In den späten 1970ern entstand das Datenbank Retrieval, das an der UB Graz 1979 eingeführt wurde. Um erste Erfahrungen mit dieser neuen Informationsmöglichkeit zu sammeln, erhielt die UB Graz bereits 1978 einen Zugang zu den Datenzentralen von Lockheed in Palo Alto (Kalifornien) sowie BLAISE in London. ${ }^{36}$ Allein der Datenbankanbieter Dialog (Lockheed, Palo Alto) umfasste damals bereits über 300 Datenbanken. Für die Recherche wählte man sich per Modem über die Telefonleitung in Palo Alto ein, dem damaligen Zentrum der Datenbankwelt. An der Universität Graz stand dafür ein 300bit-Modem zur Verfügung. Allein die UB Graz verbrauchte die Hälfte des Volumens der Bandbreite der gesamten Universität. Die andere Hälfte wurde vom Institut für Physik gebraucht, das damals bereits mit CERN ${ }^{37}$ kooperierte. 
Alle Datenbank-Abfragen liefen über das Terminal, der Zugang war nur an der UB möglich. Benutzer:innen konnten damals noch nicht selbst recherchieren, da die Suchanfragen in einer speziellen, sehr komplexen Suchsprache gestellt werden mussten und die Recherche Erfahrung im Umgang mit Retrievalsystemen erforderte. Für eine Datenbankrecherche in z.B. Dialog kamen die Benutzer:innen daher an die Bibliothek, wo sie mit einem bzw. einer Bibliothekar:in/Informationsspezialist:in die Suchanfrage besprachen. Die Suchanfrage musste dabei schriftlich auf Deutsch und auf Englisch formuliert werden, dafür gab es eigene Formulare mit Angabe der Schlagwörter, nach denen gesucht werden sollte, sowie der erwarteten Anzahl an Treffern. Dann führte der bzw. die Bibliothekar:in die Recherche durch. An der UB Graz war dafür der Informationsdienst UBIS (siehe Kap. 1.4.2.) zuständig. Die Benutzer:innen waren bei der Recherche oft anwesend. Die Suche wurde am Terminal auf einer Magnetbandkassette mitgeschrieben und gespeichert.

Die Suchanfrage selbst musste Zeile für Zeile eingegeben werden, da es noch keine grafische Oberfläche mit Suchfeldern gab. Da nur die Erstautor:innen aufgenommen wurden, war es für die Suchanfrage wichtig, diese zu kennen. Problematisch war es, wenn man den bzw. die Erstautor:in nicht wusste und so den Eintrag nicht finden konnte. Insbesondere bei Autorennamen, die Umlaute enthielten, war die Suche schwierig, denn sowohl der ASCII-Code als auch die Datenbanken, deren Entwicklung von Amerika ausging, kannten keine Umlaute.

Auf dem Bildschirm wurden nur die Titel der Treffer angezeigt. Das Ergebnis einer damaligen Datenbankrecherche war also lediglich eine Liste mit Kurzzitaten, deren Titel darauf schließen ließen, dass sie zum gesuchten Thema passen könnten. Es gab weder einen Zugriff auf die vollständigen bibliographischen Angaben noch auf die Artikel selbst. ${ }^{38}$

Die Ergebnisse der Suche konnten online über UBIS ausgedruckt werden, was allerdings teuer war, oder per Brief bestellt werden. Auch dabei handelte es sich nicht um die Volltexte, sondern lediglich um die Vollzitate mit den genauen Quellenangaben. Ein paar Tage später erhielt man per Post den Brief mit den Zitaten. Bereits die vollständige bibliographische Angabe zeigte in manchen Fällen, dass der eine oder andere Treffer doch nicht passend war. Die übrigen Artikel konnten nun in den Zeitschriftenbeständen der Bibliothek recherchiert werden. Artikel, die an der Bibliothek nicht vorhanden waren, konnten in der ÖZDB recherchiert und, sofern an einer anderen Bibliothek vorhanden, per Fernleihe kostenpflichtig bestellt werden. Auch hier stellte sich oft heraus, dass der eine oder andere Artikel nicht passend war. Auf diese Weise fielen sowohl für die Bestellung der Zitate als auch für die Fernleihe unter Umständen Kosten für Artikel an, die 
letztendlich für das gesuchte Thema nicht relevant waren. Ein erster großer Fortschritt war daher ab etwa Mitte der 1980er Jahre das Vorhandensein von Abstracts in den Ergebnissen.

Eine Datenbankrecherche war kostenintensiv ${ }^{39}$ und komplex. Neben den Kosten für die Datenbanken selbst - besonders teuer waren Businessund Patentdatenbanken - wurden die Telefonzeit, die Anschluss- sowie die Rechnerzeit der Datenbank sowie Form und Umfang der ausgegebenen Zitate verrechnet, wobei jede Datenbank eigene Preise hatte. Daher war die Angabe möglichst passender Schlagwörter sowie eine möglichst genaue Einschränkung des Themas wichtig, um rasch passende Treffer bzw. nicht zu viele Treffer zu finden. Je länger eine Suche dauerte, desto höhere Kosten fielen an. Die UB Graz übernahm für wissenschaftliche Mitarbeiter:innen und Dissertant:innen (mit Unterschrift des bzw. der Betreuer:in) die Kosten; dafür stand ein eigenes Budget zur Verfügung. Für andere Nutzer:innen war die Recherche kostenpflichtig.

An der UB Graz wurden Datenbankrecherchen insbesondere von den Mediziner:innen und in etwas geringerem Ausmaß von den Chemiker:innen benötigt. Es gab damals schon „Struktursuchen“, die von den Chemiker:innen selbst durchgeführt wurden. Die Geisteswissenschaftler:innen nutzten UBIS bzw. Datenbanken lange nicht, sondern bevorzugten die gedruckten Bibliographien.

Neben den Forscher:innen nutzten auch viele externe Firmen das Angebot einer kostenpflichtigen Datenbankrecherche, da zum damaligen Zeitpunkt viele Firmen noch keine ausreichende EDV-Ausstattung hatten und sich die teuren Datenbankzugänge auch nicht hätten leisten können. Ein Problem stellten in manchen Branchen, in denen es beispielsweise um Forschung und Entwicklung ging, die Betriebsgeheimnisse dar, da aufgrund der erforderlichen Angabe von Suchbegriffen eine Firma, die eine Recherche in Auftrag gab, bis zu einem gewissen Grad preisgeben musste, woran gerade gearbeitet wurde. Als gegen Ende der 1980er Jahre immer mehr Firmen ihre eigenen Informationsvermittlungsstellen aufbauten, gingen die Rechercheanfragen durch Firmen zurück.

\section{Die 1990er Jahre}

\subsection{CD-ROM-Datenbanken}

Die Entwicklung der CD-ROM in den 1980er Jahren brachte im Vergleich zu den bisher verwendeten Speichermedien wie Magnetbändern und Dis- 
ketten einige wesentliche Verbesserungen. Während Magnetbänder zwar eine hohe Speicherkapazität hatten, aber keine Retrieval Software mitgeliefert werden konnte, enthielten Disketten zwar eine mitgelieferte Retrieval Software, hatten jedoch nur eine geringe Speicherkapazität. Die CD-ROM verfügte sowohl über eine hohe Speicherkapazität als auch eine Retrieval Software direkt auf der CD sowie zusätzlich über standardisierte Formate für IBM- und Maclntosh-PCs ${ }^{40}$ und die Möglichkeit, zusätzliche Information in Form von Text, Audio, Video und Grafik mitzuliefern. Sie ermöglichte also die Abfrage großer Datenmengen bei gleichzeitig geringen Kosten, da für die Nutzung lediglich ein PC mit CD-Laufwerk notwendig war. Sie war daher ausgezeichnet dafür geeignet, als Speichermedium für Datenbanken zu dienen, und eine gute Alternative zu den bisherigen teuren Online-Datenbankdiensten. Bereits in den 1980er Jahren kamen daher Datenbanken auf CD-ROMs auf. Die Technologie selbst wurde ebenfalls weiterentwickelt. So kamen bald Mehrfachlaufwerke auf den Markt, die einerseits einen größeren Bedienungskomfort boten, andererseits eine notwendige Voraussetzung zur Abfrage mancher Produkte darstellten. ${ }^{41}$

Die Nutzung erfolgte entweder an Einzelplätzen oder über ein lokales Netz. In den CD-ROM-Datenbanken konnte sowohl der Standort zu einer Referenz eingespeichert und abgerufen werden, als auch direkt im System die Zusendung des Scans der Originalpublikation per Fax, Post oder Mail angefordert werden. Für die Nutzer:innen waren dies zwei wesentliche Vorteile gegenüber der bisherigen Form der Online-Datenbankrecherche. ${ }^{42}$

Ab 1988 gab es an der UB Graz die ersten lokalen CD-ROM-Datenbanken, zunächst an einem einzelnen Arbeitsplatz, zu dem bis 1991 noch drei weitere hinzukamen. ${ }^{43}$ Im Gegensatz zu den Online-Datenbanken konnten die Benutzer:innen nun erstmals selbst an den vier PCs in den CDs recherchieren. Durch die starke Auslastung der Geräte waren Terminvereinbarungen erforderlich, die Wartezeit betrug sieben bis zehn Tage. ${ }^{44}$ Die Recherche war für alle Benutzer:innengruppen kostenfrei. Die Betreuung der CD-ROM-Kund:innen übernahm nicht UBIS, sondern der Katalogdienst, da zwei (später drei) CD-ROM-Stationen im Katalograum aufgestellt waren. ${ }^{45}$

Die CDs wurden gemeinsam mit der Retrieval Software auf den PCs lokal installiert, eine Recherche war also auch bei diesen Datenbanken nur vor Ort an der Bibliothek möglich. Der Verwaltungsaufwand war hoch, denn die CDs waren natürlich nur zu dem Zeitpunkt, an dem sie gepresst wurden, aktuell, sodass in regelmäßigen Abständen von jeweils einigen Monaten neue CDs von den Verlagen zugesandt wurden.

CD-ROM-Datenbanken hatten bereits eine grafische Oberfläche mit maskenbasierter Suchsprache, die Bedienung erfolgte aber noch ohne 
Maus mittels Menüauswahl und Funktionstasten. Die unterschiedlichen Oberflächen der Datenbanken waren kein Problem, die unterschiedliche Suchsyntax allerdings schon. Ab 1992 waren beim SCl und seinem sozialwissenschaftlichen Pendant, dem Social Science Citation Index (SSCI), auch Abstracts auf den CD-ROMs.

Mit der wachsenden Beliebtheit des Mediums stieg auch die Nachfrage nach uneingeschränkten Suchmöglichkeiten unabhängig von den UB-Öffnungszeiten und der Inanspruchnahme der dort zur Verfügung stehenden PCs durch andere Benutzer:innen. Der Weg führte zu vernetzten Diensten. An der UB Graz zeigte sich bald, dass auch die vier Einzelplätze für die Nachfrage nicht ausreichten und eine Anschaffung weiterer PCs den Engpass nicht beheben würde, sondern eine Integration der Datenbanken in das Universitätsnetz erforderlich war. Die Universität Graz verfügte über ein gut ausgebautes und betreutes Ethernet, ein lokales Datennetz, an das 1991 bereits fast alle Institute angeschlossen waren. Als Lösung wurde in Zusammenarbeit von Bibliothek und EDV-Zentrum der Universität ein CDROM-Netz entwickelt, das Anfang 1992 in Betrieb ging.

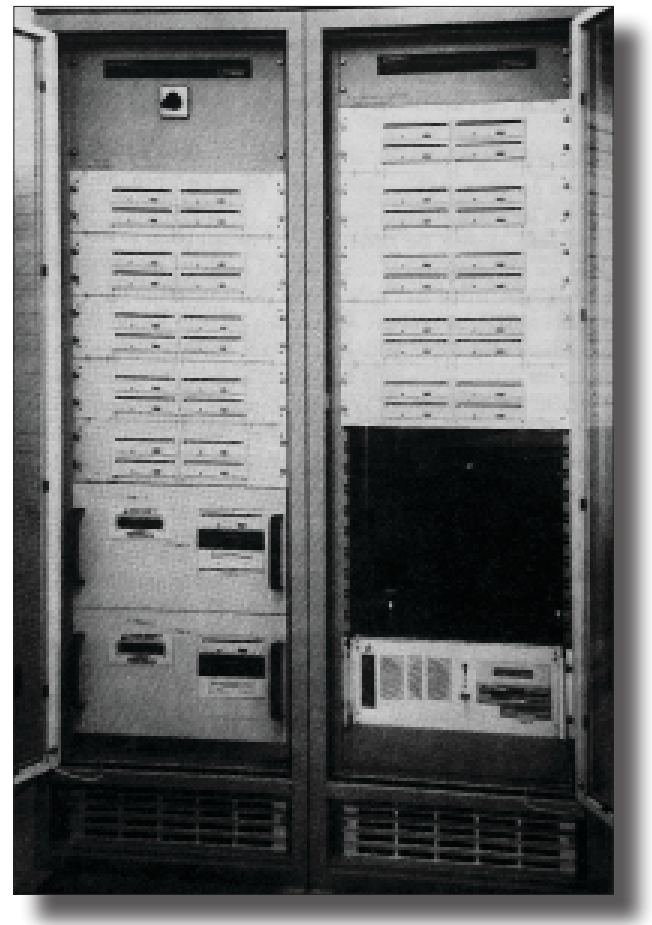

Abb. 5: CD-ROM-Server der UB Graz (Quelle: Jahresbericht der UB Graz für das Jahr 1991, S. 37). 
Das Netz bestand aus zwei Servern mit je 21 Laufwerken (siehe Abb. 5), auf die je 16 Benutzer:innen gleichzeitig zugreifen konnten, sowie einer an den Server angeschlossenen, sogenannten "Jukebox" für weitere 100 CDs kleinerer Datenbanken, für die unterschiedliche Software nötig war. Ein Greifarm holte eine CD, legte sie ein, und die Software wurde installiert. Die Server befanden sich nicht an der UB, sondern am EDV-Zentrum, und konnten vom eigenen Arbeitsplatz aus bedient werden. Für die Benutzer:innen stellte dies eine wesentliche Verbesserung gegenüber der bisherigen CD-ROM-Recherche an der Bibliothek dar, da sie sich nun direkt von ihren Instituts-PCs aus einwählen und recherchieren konnten und die Recherche unabhängig von den Öffnungszeiten der UB rund um die Uhr möglich war. ${ }^{46}$

Größere Datenbanken bestanden aus mehreren CD-ROMs, der SCI beispielsweise aus 14. Eine Suche konnte daher nicht über alle Jahrgänge hinweg erfolgen, sondern nur pro $\mathrm{CD}$, im Falle des $\mathrm{SCl}$ in Sequenzen von z.B. 5 Jahren. Die CD-ROMs wurden jedoch kontinuierlich für größere Datenmengen weiterentwickelt, sodass immer mehr Anbieter die gleichzeitige Suche über mehrere Jahrgänge hinweg ermöglichten, was ein großer Wunsch seitens der Benutzer:innen war. ${ }^{47}$

Das CD-ROM-Netz hatte trotz schlechter Performance und teils holpriger Software eine sehr hohe Akzeptanz, was der sprunghafte Anstieg der CD-ROM-Suchen nach Einführung des Netzwerks Anfang 1992 zeigt: Die Anzahl der CD-ROM-Suchen an der UB Graz stieg von 1991 auf 1992 etwa um das Zehnfache. ${ }^{48}$ Die mit Abstand meistgenutzte Datenbank war Medline, gefolgt vom SCl. ${ }^{49}$ Aufgrund der starken Nachfrage wurden bald weitere PCs angeschafft und 1994 die Gesamtkapazität des CD-ROM-Netzes ausgebaut sowie ein CD-ROM-Raum mit eigenem CD-ROM-Auskunftsdienst eingerichtet. ${ }^{50}$

Eine wesentliche Performanceverbesserung brachte eine neue CDROM-Netzsoftware, die es ermöglichte, sowohl die Retrievalsoftware als auch die Daten der CDs auf Festplatte zu speichern. Große, stark nachgefragte Datenbanken wie der SCI wurden daraufhin auf den Server gespielt, kleine, selten nachgefragte Datenbanken konnten weiterhin über die Jukebox genutzt werden. Eine eigene Software ermöglichte in weiterer Folge bei den großen Datenbanken eine Suche über alle CDs hinweg, was ein großer Fortschritt war. Auch hier war jedoch der Verwaltungsaufwand hoch, da der aktuelle Stand der Datenbanken in regelmäßigen Abständen mithilfe der von den Verlagen zugeschickten CDs auf dem zentralen Server nachgeladen werden musste.

Problematisch war die Beschaffung der nicht vor Ort vorhandenen Literatur. Während relevante Zitate rasch gefunden werden konnten, dau- 
erte die Beschaffung der Artikel per Fernleihe oft mehrere Wochen. Automatisierte Fernleihsysteme und Dokumentlieferdienste waren noch in der Testphase und verhältnismäßig teuer, Volltextdatenbanken gab es erst sehr wenige, und diese deckten nur einen sehr kurzen Zeitraum ab. ${ }^{51}$

Die UB Graz war die erste österreichische Bibliothek mit einem CDROM-Netzwerk und hatte dadurch eine Vorreiterrolle inne. Im Mai 1994 untersuchte Dipl.-Ing. Schubert anhand einer Umfrage unter den Wissenschaftler:innen der Universität Graz, ob diese die eigene Recherche in den CD-ROM-Datenbanken oder eine Recherche durch UBIS in den Online-Datenbanken bevorzugten. Die Umfrage ergab, dass die Wissenschaftler:innen die eigene Recherche gegenüber einer UBIS-Recherche bevorzugten ${ }^{52}$ - wohl auch, um nicht bekanntgeben zu müssen, woran sie gerade forschten. In der Praxis wurden Recherchen durch UBIS dennoch weiterhin genutzt, jedoch weniger in Anspruch genommen als davor.

\subsection{BIBOS}

Da GRIBS nie weiterentwickelt wurde und daher im Wesentlichen auf die Magazinsbestellung und -entlehnung beschränkt blieb, wurde der Wunsch anderer Bibliotheken nach einem kommerziellen Verbundsystem, das alle Arbeitsbereiche einer Bibliothek abdecken konnte, immer stärker. An der Sozialwissenschaftlichen Studienbibliothek der Arbeiterkammer Wien wurde in den 1980er Jahren in Zusammenarbeit mit der EDV-GesmbH, einer Wiener Firma, das zentrale Titelerfassungssystem BIBOS (Bibliotheksorganisationssystem) entwickelt, über das auch die Ausleihe abgewickelt werden konnte und das wie GRIBS ein integriertes Bibliothekssystem war. BIBOS hatte neben der Katalogisierung auch eine Erwerbungs-, jedoch keine Fernleihkomponente. Im Jahr 1982 ging BIBOS an der AK Wien in Betrieb. An der UB Graz war BIBOS 1986 für sechs Monate im Testeinsatz. 1987 erfolgte eine öffentliche Ausschreibung für die Einführung eines automationsunterstützten integrierten Verbundsystems. Von allen Bibliotheken sowie der Planungsstelle wurde ein Bewertungsschema für die Teilbereiche Katalogisierung, Geschäftsgang, Online-Publikumskatalog (OPAC) und Ausleihe ausgearbeitet. Von den Angeboten erfüllte kein System alle Anforderungen; die Wahl fiel letztendlich auf BIBOS. ${ }^{53}$ Ab 1988 wurde an der UB Graz BIBOS in einigen Teilbereichen eingesetzt (Katalogisierung Altbestand, Freihandbestand der Sondersammlungen und des Instituts für Kunstgeschichte) $)^{54}$. Im Jahr 1990 wurden die ersten Bearbeiter:innen-PCs angeschafft, während die Benutzer:innen weiterhin an Terminals Literatur suchten und bestellten. ${ }^{55} 1993$ wurden die Katalogisierung, die inhaltliche 
Erschließung und der gesamte Geschäftsgang auf BIBOS umgestellt, ${ }^{56} \mathrm{ab}$ 1994 wurden auch die Bestände der dezentralen Bereiche mittels BIBOS katalogisiert. ${ }^{57}$

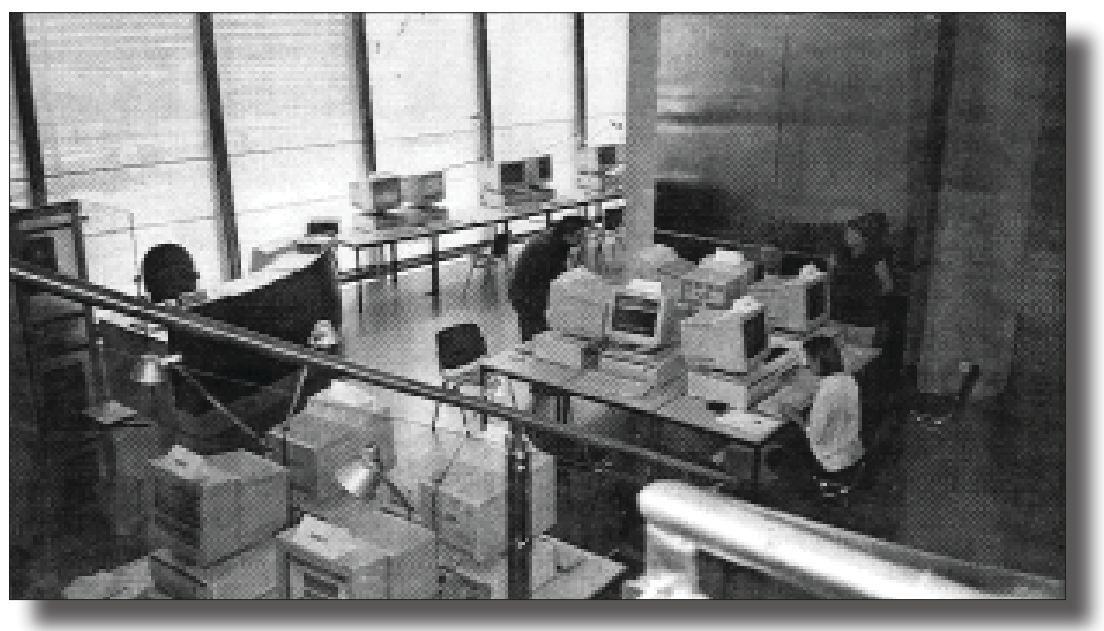

Abb. 6: Katalograum der UB Graz (Quelle: Jahresbericht der UB Graz für das Jahr 1998, S. 53).

BIBOS verfügte über eine maskenorientierte Oberfläche, was die Bedienung wesentlich erleichterte. Musste man zuvor mit einer eigenen Suchsprache Eingaben machen, so hatte man nun Felder, zwischen denen man per Tastenbefehl hin- und herspringen und dort etwas eingeben konnte. ${ }^{58}$

Die Titelerfassung in BIBOS war sehr ausgefeilt. Zum einen konnte der gesamte Titel erfasst werden, nicht nur ein Kurztitel wie in GRIBS, zum anderen konnten auch komplexe Hierarchien abgebildet und verwaltet werden. Damals fiel die Entscheidung für das „Maschinelle Austauschformat für Bibliotheken“ (MAB) und die Katalogisierung nach den „Regeln für die alphabetische Katalogisierung in wissenschaftlichen Bibliotheken" (RAKWB). Bei der Katalogisierung waren insbesondere die Bibliotheken in Innsbruck und Wien führend. Die Daten wurden über eine Standleitung an die UB Wien übermittelt. Auch die Abfrage der Daten lief über die UB Wien, was bei vielen zeitgleichen Abfragen zu Problemen führte, da die Server diese nicht schnell genug verarbeiten konnten. Aufgrund der ausgefeilten, komplexen Titelerfassung und der guten Datensätze war BIBOS bei den Katalogisierer:innen sehr beliebt und wurde in über 20 Bibliotheken eingesetzt. Das Entlehnsystem von BIBOS hatte dagegen einige Schwächen und setzte sich daher nicht durch. Die Entlehnung funktionierte zunächst per 
Standleitung über Wien, erst später wurden eigene Lokalsysteme an den einzelnen Bibliotheken eingerichtet.

An der UB Graz liefen GRIBS und BIBOS parallel. Die Nutzer:innen suchten zunächst in BIBOS, GRIBSinfo oder dem Zettelkatalog und bestellten anschließend in GRIBS. Bei den Nutzer:innen war allerdings GRIBSinfo für die Recherche wesentlich beliebter als BIBOS. Daher wurden aus den Langtiteln von BIBOS Kurztitel generiert und diese in GRIBSinfo eingespielt. Eine Datenmigration aus anderen Systemen wie GRIBS oder ÖZDB nach BIBOS war dagegen nicht möglich.

\section{Die Entwicklung seit 2000}

\subsection{Datenbanken im Internet}

Die Datenbankrecherche wanderte schrittweise ins Internet. 1995 wurde an der UB Graz mit der sukzessiven Umstellung der CD-ROM-Datenbanken auf einen Online- bzw. Internetzugang begonnen sowie eine Homepage gestaltet und auf einem eigenen Webserver eingerichtet. Die ersten beiden Datenbanken, die 1995 testweise und ab 1996 im Echtbetrieb über das Internet angeboten wurden, waren Medline und ERIC. ${ }^{59}$

Die neuen Online-Datenbanken unterschieden sich von den früheren, über Palo Alto zugänglichen Datenbanken in zweierlei Hinsicht. Zum einen besaßen sie eine grafische Oberfläche, was die Benutzung entscheidend erleichterte und die Recherche ohne komplexe Suchsprache ermöglichte. Zum anderen wurden neue Kostenmodelle angeboten, die sich im Zuge der Umstellung auf die CD-ROM-Datenbanken entwickelt hatten. War bei den alten Online-Datenbanken eine Verrechnung je nach Datenbank zu unterschiedlichen Preisen üblich gewesen (abhängig von Anschluss- und Rechnerzeit sowie Form und Umfang der ausgegebenen Zitate, was zu großen Kostenschwankungen führen konnte und sowohl die Budgetplanung als auch die Abrechnung seitens der Bibliothek erschwerte), so wurde mit den CD-ROM-Datenbanken die Verrechnung von Pauschalbeträgen eingeführt. Monatliche oder jährliche Abrechnungen der neuen Online-Datenbanken auf Basis einer Pauschale pro Zugriff waren viel leichter abrechenbar und die Kosten für die Bibliothek planbarer.

Für eine Umstellung von den CD-ROM-Datenbanken auf die neuen Online-Datenbanken waren gewisse Voraussetzungen erforderlich. Es musste eine Internet-Version der betreffenden Datenbank verfügbar sein, diese musste leistbar sein (die Online-Version war oft viel teurer als die 
CD-ROM-Version), und in den Lizenzverträgen musste eine monatliche oder jährliche Pauschalvergütung vorgesehen sein, sodass die Kosten abschätzbar waren. Mit dem Umstieg auf Online-Datenbanken wurde auch die Mausbedienung von Datenbanken eingeführt.

An der UB Graz wurde diese Umstellung sukzessive durchgeführt. Große Datenbanken boten zuerst Online-Versionen an und wurden daher zuerst umgestellt, kleine Datenbanken erst später. 2017 war die Ablösung des CDROM-Systems auf Online-Datenbanken an der UB Graz abgeschlossen.

Anfangs waren viele dieser Datenbanken nur für Bibliothekar:innen zugänglich, die daher für die Benutzer:innen Auftragsrecherchen durchführten. Doch nach und nach wurden die Datenbanken auch von zu Hause bzw. vom Institut aus zugänglich. ${ }^{60}$ Vorteile der Internet-Datenbanken waren die Tagesaktualität und die Durchsuchbarkeit des gesamten Zeitraums sowie der Zugriff über das Internet von zu Hause aus via Ultra*Net ${ }^{\mathrm{TM}}$, einer auf dem CD-ROM-Server installierten Software der Grazer Firma R+R Messtechnik Handel GesmbH, die das alte CD-ROM-System ablöste und den Zugriff von außerhalb auf den Server ermöglichte. Ab 2002 wurde neben dem Ultra*Net auch die Verbindung mittels eines virtuellen privaten Netzwerks (VPN) eingeführt, beide Zugriffsmöglichkeiten liefen viele Jahre lang parallel.

Neben der Umstellung auf Internet-Datenbanken erfolgte auch die Verknüpfung der Datenbanken mit den E-Journals mittels Linkresolvern. Ein Linkresolver stellt mittels Metadaten (beispielsweise in Form einer OpenURL ${ }^{61}$ ) und den lokalen Bestands- und Lizenzinformationen einer Bibliothek entweder eine direkte Verlinkung auf den Volltext einer wissenschaftlichen Publikation her oder leitet auf passende Dienste wie einen Dokumentenlieferdienst weiter. ${ }^{62}$ Zunächst hatte jede Datenbank ihren eigenen String. Die Entwicklung der OpenURL und damit eines normierten Suchstrings, der für die meisten Datenbanken funktionierte und die Verknüpfung von Zitat und Volltext ermöglichte, war weltweit bahnbrechend. Der SFX Linkresolver der Firma Ex Libris wurde 2001 als erster OpenURLResolver veröffentlicht. ${ }^{63}$ Die UB Graz verwendete zunächst allerdings den LinkSolver von Ovid und stieg Ende 2010 auf SFX um. Seit Sommer 2007 erfolgt der Zugriff auf die CD-ROM- und Online-Datenbanken über das Datenbank-Infosystem (DBIS). ${ }^{64}$

\subsection{Aleph}

Da GRIBS spürbar an die Grenzen seiner Leistungsfähigkeit kam, sich die Systemausfälle häuften, BIBOS nicht zweitausendfähig war und daher 
den Sprung von 1999 auf 2000 nicht geschafft hätte, benötigten die Bibliotheken rechtzeitig ein neues System. Die Arbeitsgruppe Bibliotheksautomation (AGBA) ${ }^{65}$ erhielt daher den Auftrag zur Ausschreibung eines Zentralsystems für Katalogisierung mit Lokalsystemen für Erwerbung und Entlehnung, die angeschlossen werden konnten. Zur Auswahl standen letztendlich sechs Anbieter bzw. Systeme. Von Bibliothekar:innen aus ganz Österreich wurden während oft wochenlanger Wien-Aufenthalte ausführliche Tests in mehreren Arbeitsgruppen durchgeführt und Anforderungen und Pflichtenhefte formuliert. Für die UB Graz war unter anderem Dipl.-Ing. ${ }^{\text {in }}$ Schubert im Testteam.

Die Software Aleph ${ }^{66}$ der Firma Ex Libris begeisterte mit Standards und beruhte auf einer ORACLE-Datenbank, die damals sehr verbreitet war. Allerdings gab es in Aleph keine Magazinsentlehnung. Kurzerhand programmierten die Entwickler:innen diese neue Funktion innerhalb weniger Wochen und erwiesen sich auch sonst als sehr flexibel und interessiert an der Weiterentwicklung des Systems. Dies war ein großer Pluspunkt von Aleph, denn die Betreuung und Weiterentwicklung über die AGBA war damals sehr schwierig - GRIBS wurde beispielsweise nie weiterentwickelt. Die Philosophie der Weiterentwicklung bei Aleph war daher sehr attraktiv und letztendlich ausschlaggebend für die Entscheidung. Der Zuschlag für Ex Libris erfolgte am 28. November 1997. ${ }^{67}$ Da Aleph mit MARC-Hintergrund entwickelt worden war, wurde es für den deutschsprachigen Raum auf das MAB-Format adaptiert. Das Backend für die Bearbeiter:innen war clientbasiert, das Frontend für die Bibliotheksbenutzer:innen stand als OPAC zur Verfügung.

Die Installation von Aleph erfolgte als Verbundsystem mit zentralen Komponenten (Katalogisierung, zentraler OPAC), betreut von der AGBA, sowie lokalen Komponenten (Erwerbung, Entlehnung, lokaler OPAC, lokale Katalogisierung), betreut von den jeweiligen EDV-Zentren bzw. Informatikdiensten der Universitäten sowie von den Bibliotheken. Die Fernleihe wurde erst nachträglich als zentrale sowie lokale Komponente entwickelt, wird jedoch bis heute nur in der lokalen Variante verwendet. Für die teilnehmenden Bibliotheken gab es verschiedene Optionen. So konnte ein eigenes Lokalsystem vorhanden sein, eine kleinere Bibliothek konnte ihr kleines Lokalsystem aber auch von einer größeren Bibliothek oder an der AGBA hosten lassen. Manche kleinere Bibliotheken verwendeten Alephino, die „schlankere“ Version von Aleph 500, als Lokalsystem.

Die UB Graz entschied sich für ein eigenes Lokalsystem. Zur Vorbereitung der Ablösung von BIBOS wurde an der UB Graz eine Arbeitsgruppe unter Leitung von HRin Dr. ${ }^{\text {in }}$ Böllmann und stellvertretender Leitung von 
Dipl.-Ing. in Schubert eingerichtet. Bei der Umstellung auf Aleph mussten zunächst die Titel aus BIBOS, GRIBS, FBInfo und EMILE lokal nach Aleph überspielt werden. BIBOS wurde daher mit 11. Dezember 1998 abgeschlossen, die Daten wurden nach Aleph migriert. ${ }^{68}$ Für die Migration nach Aleph schrieb eine Programmiererin des EDV-Zentrums ein Retrieval Programm, zunächst jedoch ohne die Möglichkeit, Daten nachladen zu können. Da ein Nachladen von Datensätzen jedoch notwendig war, wurde dies nachträglich angepasst. Am 11. Jänner 1999 ging die UB Graz mit Aleph 500 mit den Modulen OPAC, Geschäftsgang und Katalogisierung in Betrieb, die Entlehnung folgte im April. ${ }^{69}$ An der UB Graz löste Aleph 500 im Lauf des Jahres 1999 alle bisherigen Systeme ab: BIBOS, EMILE, FBInfo, GRIBS, GRIBSinfo und die ÖZDB. Die Benutzer:innenstammdaten wurden aus GRIBS nach Aleph transferiert und mit den E-Mail-Adressen angereichert, sodass Verständigungen über vorgemerkte, eingelangte Bücher, Erinnerungs- und Mahnschreiben seither per Mail an die Benützer:innen verschickt werden können. Im Anschluss an die Einführung des neuen Systems wurden die Mitarbeiter:innen geschult sowie Kurse für die Studierenden abgehalten.

Zum Zeitpunkt der Migration nach Aleph gab es in GRIBS noch Datensätze von Werken, die nie entlehnt und daher nie rückwirkend erfasst worden waren und daher auch keinen eingetragenen Kurztitel hatten. Da das Titelfeld ein Pflichtfeld war, wurde für jene Werke bei der Datenmigration in das Titelfeld „zzzHauptbibliothek" eingespielt. Es handelte sich um etwa 200.000 derartige Sätze. Ein Teil dieser zzz-Datensätze existiert bis heute.

Die Zeitschriften waren in GRIBS hierarchisch erfasst worden. Bei der Migration nach Aleph wurde diese Hierarchie zerschlagen, sodass jeder Band einen eigenen Titelsatz bekam. Da je nach System (GRIBS, BIBOS, Aleph) die Zeitschriftentitel entweder als Kurztitel oder vollständig erfasst worden waren, ergab sich das zusätzliche Problem unterschiedlichster Titelsätze. Im Rahmen eines eigenen Projekts wurden diese Titelsätze einige Jahre später wieder zusammengeführt.

\subsection{Vom Onlinekatalog zur Suchmaschine}

Ab Mitte der 1990er Jahre gingen die ersten kommerziellen Suchmaschinen wie AltaVista und Infoseek online, 1998 folgte Google. Die leicht zu bedienenden Suchmaschinen traten bald in immer stärkere Konkurrenz zu den Bibliothekskatalogen, den OPACs. Ein großer Vorteil der Suchmaschinen bestand in ihrer einfachen Bedienbarkeit. Für die Suche gab es lediglich ein einziges Eingabefeld, in das eine durchaus unspezifische Suchanfrage ein- 
gegeben und eine Trefferliste generiert werden konnte. Im Gegensatz dazu gab es in den OPACs eine Suchmaske mit mehreren Feldern. Die Eingabe der Suchanfrage musste bestimmten Kriterien folgen, um passende Treffer zu erhalten, daher mussten gewisse Recherchekenntnisse vorhanden sein. Die Reihung der Ergebnisse erfolgte in den Suchmaschinen mittels eines automatisierten Rankingverfahrens, während die Treffer in den OPACs nicht inhaltlich gerankt waren, sondern lediglich nach bestimmten Kriterien sortiert werden konnten (z.B. alphabetisch oder nach Erscheinungsjahr). Die relevantesten Treffer waren dadurch über die gesamte Trefferliste verteilt. Die einfache Suche in unterschiedlichsten Quellen, die inhaltliche Reihung der Ergebnisse und die umfangreichen Trefferlisten machten die Suchmaschinen rasch auch für wissenschaftliche Recherchen beliebt. Die Literaturrecherche verlagerte sich dadurch zunehmend vom klassischen Online-Bibliothekskatalog ins Internet. ${ }^{70}$

Die Weiterentwicklung der OPACs erfolgte daher auf zwei Ebenen: zum einen wurden weitere Datenbestände wie Zeitschriftenartikel, Aufsätze aus Sammelbänden und lizenzierte Datenbankinhalte über die OPACs durchsuchbar gemacht, zum anderen wurde ab den 1990er Jahren mit der Kataloganreicherung der OPACs begonnen, also der Anreicherung bibliographischer Datensätze mit weiteren Materialien wie Inhaltsverzeichnissen oder Abstracts, was einen deutlichen Mehrwert für die Benutzer:innen darstellte. ${ }^{71}$ Die UB Graz beteiligt sich seit 2007 am e-DOC-Service der OBVSG, mit dem eine Anreicherung des Verbundkataloges mit Inhaltsverzeichnissen, Abstracts, Rezensionen, Umschlagbildern, Klappentexten und, so vorhanden, Volltextdokumenten erfolgt. ${ }^{72}$

Die Durchsuchbarkeit wesentlich umfangreicherer Datenbestände führte zu deutlich umfangreicheren Trefferlisten, die mit Filtern eingeschränkt werden mussten, um möglichst passende Treffer zu bekommen. Um dem veränderten Rechercheverhalten der Nutzer:innen und dem Wunsch nach einer möglichst einfachen Literatursuche entgegenzukommen, die ähnlich einer Websuche mit gerankten Ergebnissen funktioniert, wurde versucht, Rankingfaktoren in OPACs einzuführen. Diese beschränkten sich jedoch im Wesentlichen auf text matching und Feldgewichtung (z.B. Treffer mit Suchbegriffen im Titel werden höher gereiht als Treffer mit Suchbegriffen im Abstract). ${ }^{73}$

Trotz der Weiterentwicklung der OPACs konnte das Ziel der möglichst einfachen und webähnlichen Suche nicht zufriedenstellend erreicht werden. Daher wurden so genannte Discovery-Systeme, die auf Suchmaschinentechnologie beruhten, als Alternative zum OPAC entwickelt. In Österreich wird seit 2009 das Discovery-System Primo des Anbieters Ex Libris 
eingesetzt, wobei die konsortiale Primo-Instanz an der Österreichischen Bibliothekenverbund und Service GmbH (OBVSG) angesiedelt ist. Primo kombiniert einen Rankingalgorithmus mit Filtermöglichkeiten, sodass die Treffer sowohl inhaltlich gereiht werden als auch zusätzlich durch verschiedene Filtermöglichkeiten eingeschränkt werden können.

Im Juni 2010 erwarb die UB Graz das Softwareprodukt Primo von Ex Libris und implementierte es unter dem Namen uni $\equiv$ kat. Im Februar 2011 wurde uni $\equiv$ kat in einer ersten Version in Betrieb genommen. ${ }^{74}$ In einer Übergangsphase wurden uni $\equiv$ kat und OPAC parallel betrieben. Die Umstellung vom OPAC auf Primo war für die Benutzer:innen eine große Herausforderung, da die Suche völlig anders gestaltet war und zu wesentlich mehr Treffern führte als im OPAC. Viele Benutzer:innen bevorzugten daher weiterhin den OPAC. 2012 wurde Primo Central, ein Produkt der Firma Ex Libris, als Erweiterung von uni $\equiv$ kat eingeführt, um direkt in uni $\equiv$ kat auch Inhalte von E-Journals und Datenbanken suchbar zu machen. Dafür setzte Primo Central ebenfalls SFX ein. Die Fernleihe lief noch bis Ende 2018 über den OPAC, ab Jänner 2019 konnte auch sie über Primo durchgeführt und der OPAC endgültig abgeschaltet werden. Im Herbst 2019 erfolgte im Zuge der Gestaltung eines neuen Logos die Umbenennung von uni=kat in unikat.

\section{4. $\mathrm{Alma}^{75}$}

Aleph 500 war hauptsächlich für die Verwaltung von gedruckten Werken entwickelt worden. Die Verwaltung von elektronischen Ressourcen war zwar möglich, jedoch umständlich; daher wurde nach einem neuen Bibliothekssystem für die österreichischen Verbundteilnehmer:innen gesucht. Ab Ende 2012 wurden in sechs Prüfteams die fachlichen Anforderungen an ein Nachfolgesystem erarbeitet und die in Frage kommenden Systeme getestet. Von der Abteilung Informationsdienste der UB Graz nahmen vier Personen daran teil. ${ }^{76}$

Im Oktober 2013 erfolgte die Ausschreibung, im September 2015 fiel nach einem intensiven Prüfverfahren der Einreichungen und darauffolgenden Verhandlungen die Entscheidung für die Firma Ex Libris und ihr cloudbasiertes Bibliothekssystem Alma.

Für die Umstellung der kompletten Infrastruktur des Österreichischen Bibliothekenverbundes und der an der Ausschreibung teilnehmenden Einrichtungen war ein straffer Zeitplan von zwei Jahren vorgesehen. Die 14 ausschreibenden Institutionen wurden in zwei Kohorten eingeteilt. In der ersten Implementierungsphase sollte Kohorte 1 bis September 2017 um- 
steigen, in der zweiten Implementierungsphase Kohorte 2 bis Jänner 2018, in der dritten Implementierungsphase schließlich die Verbundzentrale bis März 2018. Alle weiteren Verbundteilnehmer:innen sollten in einer mehrjährigen Übergangsphase folgen. Die UB Graz war der Kohorte 2 zugeordnet. Kohorte 1 und 2 arbeiteten während der ersten Implementierungsphase zusammen, um an kohortenübergreifenden Themen wie z.B. der Erstellung der Schulungsunterlagen zu arbeiten.

Für die Umstellung wurde sowohl eine übergreifende Projektstruktur mit Projekt- und Kernteam, OBVSG-Projektkoordination, Kohortenmanagement und lokalen Projektmanager:innen eingerichtet als auch eine lokale Projektstruktur an den jeweiligen teilnehmenden Bibliotheken, bestehend aus Projektleitung, Functional Experts für die verschiedenen Teilbereiche (u.a. Daten, E-Ressourcen, Entlehnung, Erwerbung, Fernleihe) sowie einer Schulungsgruppe.

Der Zeitplan für den Umstieg der Kohorten 1 und 2 konnte termingerecht eingehalten werden, sodass die Kohorte 2 und damit die UB Graz im Jänner 2018 den Umstieg auf das neue Bibliothekssystem Alma abschließen konnte.

\section{Fazit}

Durch den Einsatz von EDV-Bibliothekssystemen hat sich die Benutzung und Verwaltung von Bibliotheken radikal verändert. Die sehr personalintensive Entlehnung aus dem Magazin wurde durch die Einführung des ersten elektronischen Bibliothekssystems erheblich zeiteffizienter gestaltet. Die gesteigerte Benutzer:innenfreundlichkeit ließ die Entlehnungen massiv ansteigen. Mit der Weiterentwicklung der Netzwerktechnik und Übertragungsmöglichkeiten stiegen sowohl die Erwartungen an als auch die Möglichkeiten von Bibliothekssystemen.

Die Entwicklung ging von lokalen Eigenentwicklungen wie GRIBS hin zu über den österreichischen Bibliothekenverbund konsortial betriebenen Systemen wie Aleph und Alma, die von einer externen Firma weltweit vertrieben werden.

Die Entwicklung der Bibliothekssysteme prägte auch die Personalentwicklung in den Bibliotheken. Die von Bibliothekar:innen durchgeführten Suchen in Spezialdatenbanken mit speziellen Suchsprachen gingen mit der Zeit zurück, die Systeme entwickelten sich hin zu automatisierten, von den Benutzer:innen selbst getätigten Recherchen. Die Bereitstellung von Literatur in gedruckter Form und ausgewählten Datenbanken wandelte 
sich zu einer flächendeckenden Bereitstellung von Literatur in allen Medienformen, nicht zuletzt auch gestützt auf zeitgemäße Recherchemöglichkeiten. Die Betreuung der elektronischen Systeme, um die Zurverfügungstellung der Literatur sicherzustellen, schlägt sich in der Personalstruktur nieder: Systembibliothekar:innen wurden an allen Bibliotheken zum wichtigen Bestandteil des Personals.

Die Entwicklung elektronischer Datenverarbeitung an Bibliotheken ist keineswegs abgeschlossen. Neue Entwicklungen, die auf Text- und DataMining und künstlicher Intelligenz aufbauen, werden das Bibliothekswesen weiterhin wandeln und unseren Status quo ebenso veraltet aussehen lassen wie uns aus heutiger Sicht die Anfänge der EDV-Bibliothekssysteme erscheinen.

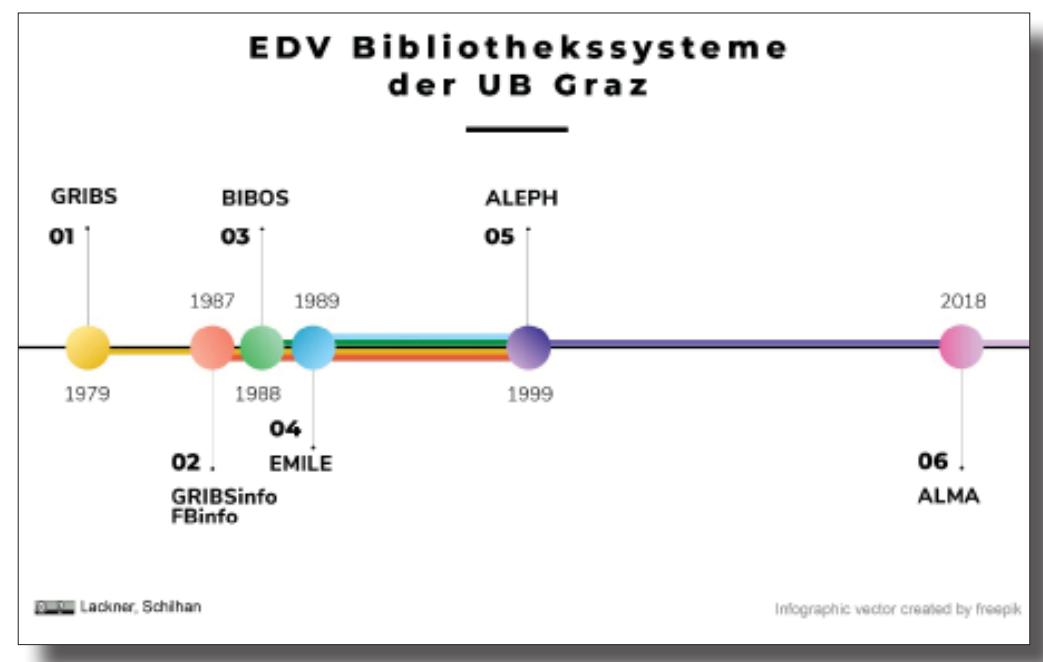

Abb. 7: Karin Lackner und Lisa Schilhan, Zeitstrahl der EDV-Bibliothekssysteme der UB Graz, CC BY 4.0

MMag. ${ }^{a}$ Karin Lackner ORCID iD: https://orcid.org/0000-0001-6096-1717 Universität Graz, Universitätsbibliothek E-Mail: karin.lackner@uni-graz.at

Mag. ${ }^{a}$ Dr. ${ }^{\text {in }}$ Lisa Schilhan ORCID iD: https://orcid.org/0000-0002-1425-850X Universität Graz, Universitätsbibliothek E-Mail: lisa.schilhan@uni-graz.at 


\section{Weiterführende Literatur}

Bruno Bauer, Markus Lackner, Bernhard Schubert (2018). Der Österreichische Bibliothekenverbund im Umbruch: Neues Bibliotheksverwaltungssystem Alma als Impuls für die Zukunft. Mitteilungen der VÖB 71(2), 260-266. https://doi.org/10.31263/voebm.v71i2.2130

Wolfgang Hamedinger (1999). Der große Wechsel: von Bibos zu Aleph 500. Mitteilungen der VÖB 52(1), 38-42. http://www.literature.at/viewer.al o? objid $=14011 \&$ viewmode $=$ fullscreen \&scale $=1.43 \&$ rotate $=$ \&page $=40$

Jahresberichte der UB Graz (ab dem Berichtsjahr 1999 online zugänglich unter https://ub.uni-graz.at/de/index.php?id=101728)

Franz Kroller (1978). Automatisierte Entlehnverbuchung an der UB Graz. Mitteilungen der VÖB 31(1), 40-42. http://www.literature.at/viewer.al o? objid=13703\&viewmode $=$ fullscreen $\&$ scale $=1.43 \&$ rotate $=\&$ page $=40$

Mitteilungen der VÖB 71 (2018) 2. Schwerpunktheft: ALMA im Österreichischen Bibliothekenverbund. https://doi.org/10.31263/voebm.v71i2 Inge Neuböck (1999). BIBOS:IV an der Sozialwissenschaftlichen Studienbibliothek der Kammer für Arbeiter und Angestellte für Wien. Mitteilungen der VÖB 52(1), 47-54. http://www.literature.at/viewer.alo?obj id $=14011 \&$ viewmode $=$ fullscreen $\&$ rotate $=\&$ scale $=1.43 \&$ page $=49$

Burghild Schubert (1988). GRIBSinfo. Mitteilungen der VÖB 41(2), Beilage Online-Mitteilungen 30, 11-17. http://www.literature.at/viewer.al o? objid=12536\&viewmode=fullscreen $\&$ scale $=3.33 \&$ rotate $=\&$ page $=13$

Gerhard Silvestri, Brigitte Schaffer (1999). Das Bibliotheksverbundsystem Bibos 1 - eine Chronologie der Jahre 1983-1998. Mitteilungen der VÖB 52(1), 55-67. http://www.literature.at/viewer.alo?objid=14011\&view mode $=$ fullscreen $\&$ scale $=3.33$ \& rotate $=$ \&page $=57$

Kurt Stubenvoll (1978). EDV-Aktivitäten an der Universitätsbibliothek Graz. Mitteilungen der VÖB 31(3), 29-31. http://www.literature.at/viewer.al o? objid $=13705 \&$ viewmode $=$ fullscreen $\&$ scale $=2 \&$ rotate $=\&$ page $=29$

Wikipedia, Bibliothekssystem: https://de.wikipedia.org/wiki/Bibliothekssystem 
1 Vgl. OCLC Online Computer Library Center (1996). The Story of the OCLC Online Union Catalog. 25th Anniversary 1971-1996, S. 1. https://library.oclc.org/digital/collection/p15003coll71/id/101/ (zuletzt abgerufen am 13.09.2021).

2 Die einzigen Ausnahmen bildeten Anfang der 1970er Jahre zwei Institutionen in Großbritannien. Erst in den 1980er Jahren gab es auch in Europa Versuche, ein Computernetzwerk aufzubauen.

3 batch job (dt. Stapelverarbeitung) ist ein Begriff aus der Datenverarbeitung. In der Anfangszeit der EDV wurden Datensätze oft in Form von Lochkarten eingelesen. Computerprogramme arbeiteten diese Lochkartenstapel (engl. batch) nacheinander ab. Der Begriff blieb bis heute als Bezeichnung für eine nicht-interaktive Datenverarbeitung erhalten, bei der vom Computer automatisiert und ohne benutzerseitigen Eingriff Aufgaben der Reihe nach abgearbeitet werden.

$4 \mathrm{Vgl}$. OCLC Online Computer Library Center (1996). The Story of the OCLC Online Union Catalog. 25th Anniversary 1971-1996, S. $3 f$. https://library.oclc.org/digital/collection/p15003coll71/id/101/ (zuletzt abgerufen am 13.09.2021).

5 ELAS wurde zwischen 1974 und 1976 entwickelt, siehe Bettina Wille (2016). Automatisierung und Digitalisierung in den wissenschaftlichen Bibliotheken der Schweiz - Ein Oral History Projekt (= Churer Schriften zur Informationswissenschaft 80), Chur, S. 25. https://www.fhgr.ch/ fileadmin/fhgr/angewandte_zukunftstechnologien/SII/churer_schriften/sii-churer_schriften_80-Automatisierung_und_Digitalisierung_in_ wissenschaftlichen_Bibliothekender_Schweiz.pdf

6 Die Planungsstelle wurde 1974 gegründet und war an der Österreichischen Nationalbibliothek (ÖNB) angesiedelt. Nachdem die Planungsstelle 1995 aufgelöst worden war, wurde im Bundesministerium für Wissenschaft, Forschung und Kunst die Arbeitsgruppe Bibliotheksautomation (AGBA) gegründet, die 2002 privatisiert wurde und nach dem Wechsel der Rechtsform seither unter dem Namen „Österreichische Bibliothekenverbund und Service GmbH“ (OBVSG) weitergeführt wird. Vgl. Website der OBVSG, https://www.obvsg.at/wir-ueber-uns/geschichte (zuletzt abgerufen am 14.09.2021).

7 Vgl. Jahresbericht der UB Graz für das Jahr 1977, S. 21.

8 Vgl. ebd., S. 21.

9 Unter anderem war Frauen damals per Gesetz Nacht- und Wochenendarbeit verboten - eigentlich ein Gesetz zum Schutz der Frauen, jedoch für viele Betriebe ein Argument, Frauen nicht im IT-Bereich einzustellen, da bei Systemarbeiten und -umstellungen, welche außerhalb der 
Betriebszeiten erfolgen mussten, Nacht- und Wochenendarbeiten unvermeidlich waren. Weibliche Angestellte konnten durch das Gesetz für diese Arbeiten nicht eingesetzt werden.

10 Vgl. Jahresbericht der UB Graz für das Jahr 1978, S. 16.

11 Für Zeitschriften mit vielen Bänden gab es einen so genannten „Spiegel", bestehend aus dem Zeitschriftentitel sowie der Angabe der vorhandenen Hefte und wie diese gebunden waren. Diese Spiegel wurden in GRIBS als Zeitschriftensignaturen mit Kurztitel, Reihenangaben und Bänden erfasst („Spiegelsignatur“). Die Zeichenanzahl war auf eine Bildschirmzeile begrenzt. Es gab Reihen und Unterreihen, die mit Sternchen darunter erfasst wurden, sowie die vorhandenen Bände. Damit man den richtigen Band bestellen konnte, musste man alle Zeilen am Terminal richtig eingeben.

12 Die genannten Kategorien wurden im Originalwortlaut übernommen und daher nicht gegendert.

13 Vgl. Jahresbericht der UB Graz für das Jahr 1984, S. 45.

$14 \mathrm{Vgl}$. Jahresbericht der UB Graz für das Jahr 1986, S. 34.

15 Vgl. Jahresbericht der UB Graz für das Jahr 1988, S. 42.

16 Vgl. Jahresbericht der UB Graz für das Jahr 1979, S. 28.

17 Die Eingliederung der Universitätsbibliotheken in die Universitäten erfolgte mit dem Universitätsgesetz 2002.

18 Vgl. Jahresbericht der UB Graz für das Jahr 1980, S. $34 f$.

19 Siehe Burghild Schubert (1980). Grazer On-Line-Ausleihe-System Magazinsbestand (Schriftenreihe EDV-Projekt 7). Graz, S. 2. 20 Siehe ebd., S. 8.

$21 \mathrm{Vgl}$. Jahresbericht der UB Graz für das Jahr 1983, S. 49.

22 Vgl. Jahresbericht der UB Graz für das Jahr 1986, S. 33.

$23 \mathrm{Vgl}$. Jahresbericht der UB Graz für das Jahr 1988, S. $42 \mathrm{f}$.

$24 \mathrm{Vgl}$. Jahresbericht der UB Graz für das Jahr 1986, S. 31.

25 Vgl. Jahresbericht der UB Graz für das Jahr 1977, S. 22.

26 Vgl. Jahresbericht der UB Graz für das Jahr 1983, S. 56.

27 Beispiel eines Kurztitels: Makatsch, W: Die Eier der Voegel Europas.

1974. (Vollständiges Zitat: Makatsch, Wolfgang, Die Eier der Vögel Europas. Eine Darstellung der Brutbiologie aller in Europa brütenden Vogelarten, Leipzig/Radebeul 1974)

28 Vgl. Jahresbericht der UB Graz für das Jahr 1987, S. 87.

29 Siehe http://emile.uni-graz.at/, Tab „Über Emile“. Zuletzt abgerufen am 13.10.2021.

30 „FB” steht für „Fachbibliothek“.

31 Vgl. Jahresbericht der UB Graz für das Jahr 1979, S. 31-33. 
$32 \mathrm{Vgl}$. Eva Bertha (2013). Bibliotheksautomation an österreichischen Universitätsbibliotheken. In: Bruno Bauer, Christian Gumpenberger, Robert Schiller (Hg.). Universitätsbibliotheken im Fokus. Aufgaben und Perspektiven der Universitätsbibliotheken an öffentlichen Universitäten in Österreich, Graz, S. 132f. (Sammelband online verfügbar unter: https://phaidra.univie.ac.at/o:376821)

$33 \mathrm{Vgl}$. Jahresbericht der UB Graz für das Jahr 1985, S. 40.

34 In den 1980er Jahren erfolgte im Bibliothekswesen die schrittweise Ablösung des Zettelkatalogs durch den elektronischen Katalog, der für die Bibliotheksbenutzer:innen an Terminals über ein lokales Netzwerk (ab den 1990er Jahren über das Internet) zugänglich war. Er wurde als „Online Public Access Catalogue“, kurz OPAC, bezeichnet. Die UB Graz hatte mit GRIBSinfo bereits eine frühe Form des OPACs.

35 Der permuterm subject index wurde seit 1966 publiziert, siehe Eugene Garfield (1984). The Permuterm Subject Index: An Autobiographical Review. In: Essays of an Information Scientist 7, 546. http://garfield. library.upenn.edu/essays/v7p546y1984.pdf

36 Vgl. Jahresbericht der UB Graz für das Jahr 1978, S. 17.

37 Europäische Organisation für Kernforschung

38 Bei manchen Datenbanken war die Übertragung der Volltexte zwar prinzipiell möglich, jedoch sehr teuer und wurde daher nicht gemacht.

39 Je nach Datenbank zwischen etwa 500 und 2.000 Schilling, vgl. Jahresbericht der UB Graz für das Jahr 1986, S. 163.

40 CDs, die standardisierte Formate für beide PC-Typen anboten, wurden als „Janus Disks" bezeichnet.

41 Vgl. Burghild Schubert, Elisabeth Böllmann (1994). CD-ROM Dienste auf dem Weg zu Mehrwertdiensten - Realitäten und Visionen. In: Wolf Rauch, Franz Strohmeier, Harald Hiller, Christian Schlögl (Hg.). Mehrwert von Information - Professionalisierung der Informationsarbeit. Proceedings des 4. Internationalen Symposiums für Informationswissenschaft (ISI 1994), Graz, 2.-4. November 1994, S. 255-256.

$42 \mathrm{Vgl}$. Eva Bertha (1994). Funktionelle Erweiterung von Bibliothekskatalogen. In: Wolf Rauch, Franz Strohmeier, Harald Hiller, Christian Schlögl (Hg.). Mehrwert von Information - Professionalisierung der Informationsarbeit. Proceedings des 4. Internationalen Symposiums für Informationswissenschaft (ISI 1994), Graz, 2.-4. November 1994, S. 221-222.

43 Vgl. Burghild Schubert, Elisabeth Böllmann (1994). CD-ROM Dienste auf dem Weg zu Mehrwertdiensten - Realitäten und Visionen. In: Wolf Rauch, Franz Strohmeier, Harald Hiller, Christian Schlögl (Hg.). Mehrwert von Information - Professionalisierung der Informationsarbeit. 
Proceedings des 4. Internationalen Symposiums für Informationswissenschaft (ISI 1994), Graz, 2.-4. November 1994, S. 256.

44 Vgl. Jahresbericht der UB Graz für das Jahr 1991, S. 31. 45 Vgl. Jahresbericht der UB Graz für das Jahr 1990, S. 45.

$46 \mathrm{Vgl}$. Burghild Schubert, Elisabeth Böllmann (1994). CD-ROM Dienste auf dem Weg zu Mehrwertdiensten - Realitäten und Visionen. In: Wolf Rauch, Franz Strohmeier, Harald Hiller, Christian Schlögl (Hg.). Mehrwert von Information - Professionalisierung der Informationsarbeit. Proceedings des 4. Internationalen Symposiums für Informationswissenschaft (ISI 1994), Graz, 2.-4. November 1994, S. 256-258.

47 Vgl. ebd., S. 258.

48 Vgl. ebd., S. 257.

49 So erfolgten beispielsweise im Jahr 1996 insgesamt etwa $25.600 \mathrm{Zu}$ griffe auf Medline sowie etwa 7.650 auf den SCI. Im Jahr 1998 wies der SCI bereits 13.000 Zugriffe auf. (Vgl. Jahresbericht der UB Graz für das Jahr 1996, S. 61, sowie für das Jahr 1998, S. 70).

$50 \mathrm{Vgl}$ Jahresbericht der UB Graz für das Jahr 1994, S. $34 \mathrm{f}$.

$51 \mathrm{Vgl}$. Burghild Schubert, Elisabeth Böllmann (1994). CD-ROM Dienste auf dem Weg zu Mehrwertdiensten - Realitäten und Visionen. In: Wolf Rauch, Franz Strohmeier, Harald Hiller, Christian Schlögl (Hg.). Mehrwert von Information - Professionalisierung der Informationsarbeit. Proceedings des 4. Internationalen Symposiums für Informationswissenschaft (ISI 1994), Graz, 2.-4. November 1994, S. 259.

52 Zur detaillierten Auswertung der Umfrage siehe ebd., S. 255-260.

$53 \mathrm{Vgl}$. Jahresbericht der UB Graz für das Jahr 1987, S. 32.

$54 \mathrm{Vgl}$. Jahresbericht der UB Graz für das Jahr 1989, S. 35.

55 Vgl. Jahresbericht der UB Graz für das Jahr 1990, S. 41.

56 Vgl. Jahresbericht der UB Graz für das Jahr 1993, S. 29.

$57 \mathrm{Vgl}$. Jahresbericht der UB Graz für das Jahr 1994, S. 34.

58 Die Entwicklung einer grafischen Benutzeroberfläche, dem so genannten Graphical User Interface (GUI), erfolgte erst ab 1992, u.a. mit Netscape. $59 \mathrm{Vgl}$. Jahresbericht der UB Graz für das Jahr 1995, S. 45.

60 Heute gibt es nur noch sehr wenige Datenbanken, auf die ausschließlich von Bibliothekar:innen zugegriffen werden kann.

61 siehe https://de.wikipedia.org/wiki/OpenURL.

62 siehe https://de.wikipedia.org/wiki/Linkresolver.

63 siehe https://exlibrisgroup.com/de/produkte/primo/sfx-link-resolver/. 64 Vgl. Jahresbericht der UB Graz für das Jahr 2007, S. 37.

65 Die AGBA wurde 1995 aus der Planungsstelle für wissenschaftliches Bibliothekswesen aus- und in das Bundesministerium für Wissenschaft 
und Forschung eingegliedert. Nachfolgerin ist die 2002 gegründete Österreichische Bibliothekenverbund und Service GmbH (OBVSG).

66 Aleph wurde an der Hebräischen Universität Jerusalem entwickelt und ging dort erstmals 1981 als Aleph 300 in Betrieb. Weltweit vertrieben wird Aleph von der Firma Ex Libris.

67 Vgl. Jahresbericht der UB Graz für das Jahr 1998, S. 49.

68 Vgl. ebd., S. 49.

69 Siehe Jahresbericht der UB Graz für das Jahr 1999, S. 55 u. 57.

70 Vgl. Dirk Lewandowski (2010). Der OPAC als Suchmaschine. In: Julia Bergmann and Patrick Danowski (Hg.). Handbuch Bibliothek 2.0, S. 87-107. https://doi.org/10.1515/9783110232103.87

$71 \mathrm{Vgl}$. ebd.

72 Vgl. Jahresbericht der UB Graz für das Jahr 2007, S. $36 f$.

73 Vgl. Dirk Lewandowski (2010). Der OPAC als Suchmaschine. In: Julia Bergmann and Patrick Danowski (Hg.). Handbuch Bibliothek 2.0, S. 87-107. https://doi.org/10.1515/9783110232103.87

$74 \mathrm{Vgl}$. Jahresbericht der UB Graz für das Jahr 2011, S. 30.

75 Soweit nicht anders angegeben, stammen die Informationen in diesem Kapitel aus: Markus Lackner (2018). Die Alma-Implementierung im österreichischen Bibliothekenverbund (OBV) unter besonderer Berücksichtigung der Kohorte 2-Bibliotheken. Mitteilungen der VÖB 71(2), S. 267-275. https://doi.org/10.31263/voebm.v71i2.2131

76 Vgl. Jahresbericht der UB Graz für das Jahr 2012, S. 29. 